Draft Version SEPTEMber 13, 2018

Preprint typeset using $\mathrm{LAT}_{\mathrm{E} X}$ style emulateapj v. 6/22/04

\title{
FOURIER DISSECTION OF EARLY-TYPE GALAXY BARS
}

\author{
R. Buta ${ }^{1}$, E. Laurikainen ${ }^{2}$, H. Salo ${ }^{2}$, D. L. Block ${ }^{3}$, And J. H. Knapen ${ }^{4}$ \\ Draft version September 13, 2018
}

\begin{abstract}
This paper reports on a near-infrared survey of early-type galaxies designed to provide information on bar strengths, bulges, disks, and bar parameters in a statistically well-defined sample of S0-Sa galaxies. Early-type galaxies have the advantage that their bars are relatively free of the effects of dust, star formation, and spiral structure that complicate bar studies in later type galaxies. We describe the survey and present results on detailed analysis of the relative Fourier intensity amplitudes of bars in 26 early-type galaxies. We also evaluate the symmetry assumption of these amplitudes with radius, used recently for bar-spiral separation in later-type galaxies.

The results show a wide variety of radial Fourier profiles of bars, ranging from simple symmetric profiles that can be represented in terms of a single gaussian component, to both symmetric and asymmetric profiles that can be represented by two overlapping gaussian components. More complicated profiles than these are also found, often due to multiple bar-like features including extended ovals or lenses. Based on the gravitational bar torque indicator $Q_{b}$, double-gaussian bars are stronger on average than single-gaussian bars, at least for our small sample. We show that published numerical simulations where the bar transfers a large amount of angular momentum to the halo can account for many of the observed profiles. The range of possibilities encountered in models seems well-represented in the observed systems.

Subject headings: galaxies: spiral; galaxies: photometry; galaxies: kinematics and dynamics; galaxies: structure
\end{abstract}

\section{INTRODUCTION}

S0 galaxies were introduced to the Hubble sequence by Hubble (1936) as a means of bridging the apparently catastrophic gap between E7 and Sa galaxies. After real examples were discovered (see Sandage 1961), the hallmark of the class became a disk shape (definitely "later than" E6 or E7) and an absence of spiral arms or star formation. SB0 galaxies were originally classified as SBa by Hubble (1926) even though they also lacked arms. This inconsistency was corrected in Sandage (1961).

Barred S0 galaxies are extremely interesting because they help to take some of the mystery out of S0s in general: a bar is usually a disk feature that is closely related to spiral structure in many galaxies (e.g., Kormendy and Norman 1979). In addition, ring features are directly related to bars in spirals, and SB0s may show vestiges of similar rings. One could therefore ask whether bar properties in S0s might provide any clues as to how S0s and spirals might be related in an evolutionary sense.

We are interested in the distribution of bar strengths in S0 galaxies, a property of very early-type galaxies that has not yet been tapped for what it might tell us about the evolutionary history of S0 galaxies. Early-type galaxies have a remarkable array of bar morphologies whose Fourier and other properties are worth characterizing in more detail. The advantage we have for examining these issues is that early-type galaxies show their bars largely

\footnotetext{
${ }^{1}$ Department of Physics and Astronomy, University of Alabama, Box 870324, Tuscaloosa, AL 35487

${ }^{2}$ Division of Astronomy, Department of Physical Sciences, University of Oulu, Oulu, FIN-90014, Finland

3 School of Computational and Applied Mathematics, University of the Witwatersrand, Johannesburg, South Africa

${ }_{4}$ Centre for Astrophysics Research, University of Hertfordshire, Hatfield AL10 9AB, UK
}

unaffected by dust, star formation, and spiral structure. We can look for subtle structural differences between different bars and isolate possible different bar types.

We are also interested in comparing early-type barred galaxies with the models of Athanassoula (2003, 2005), who demonstrated the importance of angular momentum transfer to the halo as a means of producing strong bars. The Fourier profile information we provide here is ideally suited to comparison with $n$-body models, and for evaluating the symmetry assumption used for bar-spiral separation (Buta, Block, \& Knapen 2003).

The Near-InfraRed S0 Survey (NIRS0S) is an attempt to obtain a statistically well-defined database of images of S0s from which the properties of S0 bars may be fairly compared to those of spirals. The Ohio State University Bright Galaxy Survey (OSUBGS, Eskridge et al. 2002) provides a valuable dataset for studying the properties of spiral galaxies, and has been fully tapped for bar strength studies by Block et al. (2002), Buta, Laurikainen, \& Salo (2004=BLS04), Laurikainen, Salo, \& Buta (2004a), Laurikainen et al. (2004b), and Buta et al. (2005). The questions we address with the NIRS0S sample are: (1) how strong do S0 bars get compared to spiral bars? (2) how does the distribution of bar strengths in $\mathrm{S} 0$ galaxies compare with that for spirals? (3) what characterizes the morphology of bars in S0 galaxies? and (4) what are the near-IR luminosity ratios and profile characteristics of bulges in $\mathrm{S} 0$ galaxies?

In describing early-type disk galaxies, we will use specific terminology. An "ansae" bar is one showing bright enhancements near the ends (Sandage 1961). A "regular" bar does not show such enhancements. Ansae bars are preferentially found in early-type galaxies while regular bars are preferentially found in later types, although no statistical study has quantified the difference. A lens 
is a feature showing a shallow brightness gradient interior to a sharp edge (Kormendy 1979). An oval is a broad elongation in the light distribution; it differs from a conventional bar in lacking higher-order Fourier terms. If intrinsically elongated, a lens can also be an oval. Ovals are discussed further by Kormendy and Kennicutt (2004).

In this paper, we focus on a set of 26 galaxies from the NIRS0S having ovals and/or bars, and investigate radial profiles of relative Fourier intensity amplitudes. We seek to examine the diversity in early-type bars according to the symmetry of such profiles.

In section 2, we describe first the rationale for the survey and the sample selection criteria. In section 3 , we describe the observations made with different instruments and detectors. The study of the relative Fourier intensity amplitudes is presented in section 5. A discussion of the results is presented in section 6 .

\section{SAMPLE CHARACTERISTICS AND NATURE OF THE SURVEY}

The sample for the NIRS0S has been drawn from the Third Reference Catalog of Bright Galaxies (RC3, de Vaucouleurs et al. 1991). We selected all galaxies in the revised type range $-3 \leq \mathrm{T}<2\left(\mathrm{~S}^{-}\right.$to $\left.\mathrm{Sa}\right)$ having $B_{T} \leq 12.5$ and $\log \mathrm{R}_{25} \leq 0 . \overline{35}$ as targets for the survey, which amounts to 170 galaxies. However, any sample of S0 galaxies is bound to have a bias in the sense that some nonbarred S0s lacking any distinct structure will likely be classified as elliptical galaxies. This could have serious effects on a bar strength study. To reduce the bias somewhat, we have supplemented the sample with 20 more galaxies that are classified as $\mathrm{E}_{\text {or }} \mathrm{E}^{+}$in $\mathrm{RC} 3$, but which are shown by Sandage and Bedke (1994=SB94) to be S0 galaxies. In these cases, we believe the SB94 types to be more accurate. Related to this issue is the possibility that some galaxies classified as $\mathrm{S} 0$ in $\mathrm{RC} 3$ are actually E galaxies. This is shown to be the case with two of our ESO sample galaxies (Laurikainen et al. 2006). In order to identify such cases, we have to rely on the multicomponent decompositions.

Another potential problem in an early-type disk galaxy sample is the misclassification of edge-on disks as bars, something which could happen if an edge-on disk is immersed in a significant bulge component or if the disk is a polar ring. Detailed analysis should identify such cases.

The sample selection criteria do not perfectly match those of the OSUBGS because S0s are not as abundant in RC3 as are spirals for the OSUBGS selection criteria (mainly $B_{T} \leq 12.0$ ). We decided it was more important to try and match the sample size of the OSUBGS rather than the exact selection criteria. Although the OSUBGS had no restriction on $\log R_{25}$, we imposed such a restriction on our S0 sample because bar strengths cannot be derived reliably for highly-inclined galaxies.

The rationale for observing S0s in the near-IR requires some discussion. Although both S0's and spirals are highly flattened disk-shaped systems, S0's mostly lack spiral structure and star formation. By default such systems tend also to be low in dust content. This means that, in principle, bar strengths in S0s could be derived in optical bands with little impact due to dust. The reason we chose not to follow such an approach is that we wanted to make a fair comparison between bars in S0s and bars in spirals. In optical bands, bars in spirals may be affected by star formation, leading dust lanes, and even more disorganized dust lanes, such that the kind of analysis we describe here is less effective for determining bar properties. These effects are minimized or eliminated in the near-IR, and thus to make the comparison with spirals fair, it is essential that the S0s also be observed in the near-IR. In addition, some S0s do have dust, and the impact of this dust is minimized in the near-IR passbands.

Figure 1] shows how our sample galaxies are distributed in type, family, and absolute $B$-band magnitude. Figure 1 a shows that the dominant type is $T=-2$, or $\mathrm{S}^{\circ}$. A significant fraction of these has no family estimate in RC3, but among the ones that do, those classified as type $\mathrm{SA} 0^{\circ}$ contribute the most to the $T=-2$ peak. The unusual distribution for the SAB galaxies in the sample is partly due to classification uncertainties, as noted by de Vaucouleurs (1963). SB galaxies in the sample are more uniformly distributed across the type bins.

Table 1 summarizes mean absolute $B$-band magnitudes and RC3 types for the same samples as illustrated in Figure 1 The average absolute magnitude for the full sample is comparable to that for the spiral sample used by BLS04. Most noteworthy is that the SB galaxies in the NIRS0S sample have a fainter average absolute magnitude than the SA and SAB galaxies, the difference being significant at the $2 \sigma$ level. Also, the SAB and SB galaxies are on average of later type than the SA galaxies $(3 \sigma$ level) and for the full sample, which includes some E galaxies. The sample is still biased in much the same way as the OSUBGS sample of spirals (BLS04), with few galaxies having $M_{B}>-18$.

\section{OBSERVATIONS}

Observations related to NIRS0S have been taken at the Nordic 2.5m Optical Telescope (NOT) in January and September 2003 and January 2004, and with the ESO 3.5-m New Technology Telescope (NTT) in December 2004. The NOT observations were made with NOTCam, a multi-mode instrument that can provide images in the $1-2.5 \mu \mathrm{m}$ region. We used it to observe 45 galaxies in wide field imaging mode, which provided a $4^{\prime} \times 4^{\prime}$ field of view with 0 "' 23 pixels. Flat fielding was accomplished with twilight frames. All of the NOT galaxies were observed in the $2.15 \mu \mathrm{m} K$-short $\left(K_{s}\right)$ band, whose shorter wavelength cutoff than the regular $K$-band reduces some of the thermal background component. (See Persson et al. 1998 for a discussion of this filter and its relation to the regular Johnson $K$ filter.) In some cases, we also observed an object in the $J$-band $(1.25 \mu \mathrm{m})$ in order to use color gradients to evaluate the constancy of the stellar mass-to-light ratio used in gravitational torque analyses (Quillen, Frogel, \& González 1994). Other aspects of the data reduction are described by Laurikainen et al. $(2005=\mathrm{LSB} 05$, paper 1$)$.

The NTT observations were made with SofI, a widefield IR spectro-imager designed for the $1-2.5 \mu \mathrm{m}$ region. We used SofI to image 15 galaxies with a field of view of $4^{\prime} .9 \times 4^{\prime} .9$ and a pixel size of 0.29 . As with NOTCam, the SofI observations were made with a $K_{s}$ filter supplemented in a few cases with a $J$-band filter. The reductions for this dataset are described by Laurikainen et al. (2006, paper 3).

The galaxies observed up to the time of the present 
analysis were selected by observing conditions and the time of year, and should be a representative subset of the whole NIRS0S. A few galaxies outside the NIRSOS sample are included in the present data set because some of our NOT runs were carried out under precarious conditions of wind and seeing that limited our access to NIRS0S sample galaxies. In this circumstance, we relaxed our magnitude limit somewhat to accomodate other accessible objects.

\section{MULTI-COMPONENT DECOMPOSITIONS AND DEPROJECTED IMAGES}

LSB05 have analyzed 24 of the NOT galaxies observed for our survey. A decomposition analysis of the ESO sample is provided in a separate paper (Laurikainen et al. 2006). In addition to providing new information on the bulge and disk properties of S0s, these studies also have provided deprojected images, constructed using orientation parameters based, when possible, on deep optical images. The deprojected images are corrected for bulge deprojection stretch by using the decompositions to remove the bulge, deprojecting the disk alone, and then adding back the bulge as a spherical component. The decompositions were essential because relative Fourier intensity profiles would be affected if bulge shapes were ignored. With corrected images, we can reliably analyze these profiles for as much intrinsic information as possible. LSB05 and Laurikainen et al. (2006) list the orientation parameters used for our deprojections.

Note that in some cases, the deprojection procedure fails because the bulge has a flattening intermediate between a sphere and a highly flattened disk. This is manifested as undercorrected light along the galaxy minor axis, leaving zones deficient in light that distort the inner parts of the bar. For our analysis here, we do not use those galaxies (NGC 1350, 3626, 4340) in our present sample where these deficient zones appeared to be significant. For one of these cases (NGC 1079), we used deprojected images where the bulge is assumed to be as flat as the disk. This left some "deprojection stretch" amplitude in the inner regions but had little effect on the bar. Also, those galaxies having little or no bar are not included. This left 26 galaxies for our Fourier study.

Table 2 summarizes types, diameters, distance moduli (Tully 1988), and absolute $B$-band magnitudes of these 26 galaxies. Unlike the types used in Table 1 and Figure 11 these are new classifications taken mostly from the de Vaucouleurs Atlas of Galaxies (Buta, Corwin, and Odewahn 2006), and are based on deep optical CCD images with the exceptions of NGC 1440, 2781, 2787, and 3941 whose types Buta has estimated from photographs in Sandage and Bedke (1994). The classifications are in a modified version of the de Vaucouleurs (1959) revised Hubble-Sandage system (Buta 1995). We use these classifications here because some are more accurate than RC3 classifications. Several galaxies in Table 2 which are classified as types $\mathrm{S}^{\circ}$ or $\mathrm{S}^{+}$in RC3 are actually early-type spirals. The average revised $T$ index of the 26 galaxies on the RC3 numerical scale is -0.3 , or type S0/a. Thus, this particular subset of the NIRSOS sample is dominated by early-type transitional spirals.

\section{RELATIVE FOURIER INTENSITY PROFILES OF SB0-SBA BARS}

The NIRS0S database as of this writing does not have enough galaxies to reliably tell us about the distribution of bar strengths in early-type disk galaxies, but the sample is large enough to allow us to investigate a simple characteristic of SB0 bars: the behavior of their relative Fourier intensity amplitudes and phases with radius. This is an important issue because (1) only for early-type galaxies can the Fourier properties of bars be studied largely unaffected by spiral structure; (2) the resulting Fourier profiles have the potential for allowing us to categorize bars with different Fourier characteristics; and (3) the same kind of analysis can be applied to $n$-body model bars (e.g., Athanassoula 2003).

A particular question we ask in this paper is: Do SB0 galaxies support the symmetry assumption used in barspiral separation? That is, do the relative Fourier intensity amplitudes of all even $m$ terms decline radially past a maximum in the same or a similar manner as they rose to that maximum? Buta, Block, \& Knapen $(2003=\mathrm{BBK})$ showed that bars can be effectively separated from spirals using this kind of assumption. With the symmetry assumption, bars can be extrapolated into spiral-dominated regions, and removed from an image. If one adds the axisymmetric background to the mapped bar image, then the strength of the bar alone can be estimated without the influence of spiral torques. Using this approach, Buta et al. (2005) were able to derive the distribution of true bar strengths (rather than total nonaxisymmetry strengths) for nearly 150 galaxies in the OSUBGS. Although the symmetry assumption had some support in previous optical studies of barred galaxies (e.g., Ohta, Hamabe, \& Wakamatsu 1990), it can only be reliably evaluated if a bar is clean, i.e., not affected by dust or spiral structure. The NIRSOS is therefore ideal for validating this approach.

Our subset of 26 NIRSOS galaxies includes only those observed so far having conspicuous bars or ovals. The deprojected $K_{s}$-band images of these galaxies are shown in Figure 2 These are displayed mainly to reveal the primary bars and ovals, but not necessarily secondary bars or ovals, the discussion of which is covered in more detail in papers 1 and 3 (LSB05; Laurikainen et al. 2006). We find a variety of Fourier properties of bars. Our main result is that the relative Fourier intensity profiles of bars in SB0 galaxies can be represented in simple ways, ranging from symmetric single component bars, to symmetric multicomponent bars, to asymmetric multicomponent bars. We discuss the implications of these findings for the bar-halo interaction scenario proposed by Athanassoula (2003, 2005).

\subsection{Fourier Profiles}

Figure 3 shows plots of the relative Fourier intensities $I_{m} / I_{0}$ and phases $\phi_{m}$ for our subset of 26 NIRS0S galaxies. Although we computed these profiles for all even Fourier terms to $m=20$, Figure 3] shows only the $I_{m} / I_{0}$ profiles for $m=2,4,6$, and 8 and $\phi_{m}$ profiles for $m$ $=2$ and 4 . The latter sometimes show sharp changes in phase due merely to the $360^{\circ} / \mathrm{m}$ periodicity of the terms.

We can make a few general observations concerning these profiles. First, considering that these are near-IR images of early-type disk galaxies, the profiles of many are surprisingly complex. Second, more than $2 / 3$ of the galaxies show small peaks in $m=2$ amplitude near the 
center that are in some cases weak nuclear ovals or secondary bars. These features are almost never aligned with the primary bar according to the phase plots. Third, the $m=2$ profiles are often more complicated than the $m$ $>2$ profiles, showing extra components in the nonaxisymmetric light distribution.

Interpreting the nuclear features requires some caution. The Fourier profiles are derived from deprojected images where the bulge stretch has been corrected, but only to the extent of approximating the bulge as a spherically symmetric light distribution. In the final fits of the decompositions described in LSB05 and Laurikainen et al. (2006), most of the bulges were described by elongated light distributions. Thus, the reliability of the $m=2$ profiles and phases for the identification of central components is questionable in some cases. Although we analyze the secondary bars in NGC 1317 and 3081 in the present paper, we refer to LSB05 and Laurikainen et al. (2006) for detailed discussions of the other nuclear features.

Most notable in the Fourier plots are the galaxies whose bar profiles are exceptionally symmetric. Cases like NGC 936, 1326, 1387, 1440, 1533, 1574, 2217, 2787, $2983,3941,4596$, and 4608 have primary bars that are symmetric enough that if these bars coexisted with a spiral, the symmetry assumption would recover the bar reliably (section 5.7).

The remaining galaxies show more complicated profiles with evidence for multiple components. In some cases, these components almost do not overlap, as in NGC 1317, $1574,2681,2781$, and 3081 . In each of these cases there is an inner component whose $I_{m} / I_{0}$ profiles are symmetric, but whose outer profiles may or may not be symmetric. Other galaxies simply have asymmetric profiles for a variety of reasons. In the next section, we attempt to interpret the structure of each bar in the sample.

\subsection{Gaussian Components}

The appearance of many of the profiles in Figure 3 suggests that single or multiple gaussians in fixed position angles could represent the relative Fourier intensity profiles of bars. This provides a convenient way of specifying the radial behavior of bars that could be useful for comparisons with $n$-body simulations. Figure 4 shows our gaussian fits to all the main profiles in the sample. These were obtained via nonlinear least squares using several routines from Numerical Recipes (Press et al. 1986). In addition, Figure [5] shows reconstructed images of the mapped bars (excluding the $m=0$ term), while Figure 6 shows the light remaining after the bar model and the $m=0$ term are subtracted. The appearance of these plots depends on how many terms were needed to represent the bar. Weak bars or ovals required only a few terms, while strong bars were mapped with all even terms to $m=20$. The residual images include all odd Fourier terms as well as any even terms not part of the mapped bar. The bar mappings use average phases for each term over a range of radii.

\subsubsection{Single-Gaussian Fourier Profiles}

Figure 4 shows that some observed bars have $I_{m} / I_{0}$ profiles that are well-fitted by a single gaussian in all terms, with little or no amplitude outside this component. The bars seen in NGC 1387, 1440, 1533, 2787, and 3941 are like this. Related are cases like NGC 1574 and
2681, where an extended oval is both misaligned with and mostly outside the main bar. In these cases, the single gaussian characteristic still applies to the primary bar, which is mostly separate from the oval. NGC 2217 may also be in this category, although it has a significant secondary bar and a faint outer ring, and its profiles are less regular in appearance.

For these galaxies, the bar plus background disk/bulge light can be described mathematically as

$$
I(r, \phi)=I_{0}(r)\left[1+\sum_{m=2}^{4,6, e t c .} A_{m} e^{-\frac{\left(r-r_{m}\right)^{2}}{2 \sigma_{m}^{2}}} \cos m\left(\phi-\phi_{m}\right)\right] 1
$$

where $A_{m}$ is a constant, $r_{m}$ is the mean radius, $\sigma_{m}$ is the gaussian width, and $\phi_{m}$ is the phase for each Fourier component $m$. The parameters of these single gaussian representations are summarized in Table 3; for NGC 1317 and 3081, the fits refer to a strong secondary bar.

\subsubsection{Double-Gaussian Fourier Profiles}

Other galaxies in our sample have lower-order $I_{m} / I_{0}$ profiles that are better represented by a double gaussian fit. The bars seen in NGC 936, 1452, 2983, 4245, 4596, 4608 , and 4643 show this characteristic. For NGC 936 and 2983 , only the $m=2$ term required a double-gaussian fit, while for NGC 1452 and 4643, even the higher-order terms required double-gaussian fits. For these galaxies, the bar plus background disk light can be described mathematically as

$I(r, \phi)=I_{0}(r)\left[1+\sum_{n=1}^{2} \sum_{m=2}^{4,6, e t c .} A_{n m} e^{-\frac{\left(r-r_{n m}\right)^{2}}{2 \sigma_{n m}^{2}}} \cos m\left(\phi-\phi_{m}\right)\right]$

In principle, $\phi_{m}$ could be different for the two gaussian components, but in such a case the two features would not be part of the same bar and would have to be analyzed separately. The parameters of the double-gaussian representations are also summarized in Table 3, divided into components 1 and 2.

\subsection{Multi-Component Fourier Profiles}

The remaining galaxies in our sample show more complicated Fourier profiles compared to the single and double gaussian cases. The presence of rings, multiple bars, extended ovals, and sometimes faint spirals is often the reason why the more simple representations outlined above do not work. Nevertheless, the profiles in these cases can usually be partly interpreted in terms of single or double gaussians, as shown in Figure 3

For example, in NGC 718 the bar is an unusual multicomponent type including an inner pointy oval, two bright ansae beyond the ends of this bar, and an extended oval, all in approximately the same position angle. The double gaussian fit shown in Figure 4 does not include the extended oval. Beyond a radius of $35^{\prime \prime}$, faint spiral structure also adds amplitude.

In NGC 1022, the bar shows considerable isophote twisting. We can identify at least two components in this bar in different position angles, and show an approximate separation in Figure 4 based only on the symmetry assumption and not on gaussian fits. There is also an outer ring and weak spiral structure outside the bar region. 
The primary feature in NGC 1302 is a pointy oval that can be characterized in terms of a double gaussian with the two components in slightly different position angles. There is extra $m=2$ and 4 amplitude outside this bar that is very faint and difficult to interpret.

NGC 1317 is an interesting case where the primary feature is an oval, while the secondary bar is a normal bar in a position angle offset by $90^{\circ}$. The primary oval shows a relatively symmetric multi-component $m=2$ profile, while the secondary bar is approximately a singlegaussian type that does not appear to overlap the primary oval. The two components show Fourier profiles that are completely separated.

All of the amplitude from $10^{\prime \prime}-80^{\prime \prime}$ has virtually the same phase in NGC 1326. Its nonaxisymmetry is characterized by an ansae type primary bar imbedded within a highly elongated oval. The Fourier profiles can be represented well with a double gaussian fit to the $m=2,4$, and 6 terms. The asymmetry in each of these terms changes because the outer Fourier component weakens relative to the inner one with increasing $m$. There is extra $m=2$ and 4 light that appears to be due to enhancements in the outer ring along the bar axis.

NGC 1512 is an early-type spiral with a strong primary bar and an elongated inner pseudoring around the bar. The presence of the ring means we cannot study the bar profile alone. Figure 4 shows how we would map the bar using the symmetry assumption. The doublehumped mapping completely removes the bar from the image. The remaining amplitude is connected with the oval intrinsic shape of the inner pseudoring.

NGC 2273 is an early-type spiral having a well-defined bar, inner spiral pseudoring, and an extended oval, all in approximately the same position angle. The doublegaussian representation shown in Figure 4 provides a good approximation to the bar. The excess $m=2$ amplitude beyond $r=40^{\prime \prime}$ is due to the innermost of the galaxy's two outer rings. This ring is intrinsically aligned perpendicular to the bar and oval.

As already noted, the classified bar in NGC 2681 is mostly a single gaussian type, but outside this bar is a significantly extended and somewhat pointy oval in a very different position angle. This oval is a major feature of the galaxy's nonaxisymmetry distribution.

The $m=2$ profile of NGC 2781 shows an approximately gaussian peak at $r=66^{\prime \prime} 5$ due to a small inner disk in the center, noted by LSB05. Completely separate from this feature is a broad, extended oval with a slightly asymmetric profile. This feature can be well-represented by two displaced gaussians in slightly different position angles. The two gaussians are shown separately in Figure 4

NGC 2859 is very similar to NGC 2781 in having a very broad $m=2$ hump at larger radii. The broad hump includes a significant ansae-type bar and an extended oval. Figure 4 shows a double-gaussian representation of the two features. Significant $m>2$ amplitude is found mainly for the inner gaussian component.

NGC 3081 is the most interesting of the multicomponent cases. One can identify three clear bar-like features: a secondary bar, a weak primary bar, and an extended oval in the form of a bright elliptical inner ring (Buta \& Purcell 1998). The primary and secondary bars have nearly the same maximum value of $I_{2} / I_{0}$, but the extended oval ring is clearly the dominant nonaxisym- metric feature. The secondary bar is well-represented as a single gaussian type.

\subsection{Summary of Bar Components}

Table 4 summarizes the results of the previous section. It lists the principal feature seen in the Fourier profiles and any bar-like features seen at larger radii. The information listed in column 3 of Table 4 shows that ansaetype bars are as frequent as normal ("regular") bars in this sample. Some of the barred properties are highlighted in LSB05 and Laurikainen et al. (2006), as well as in other publications (e.g., Wozniak et al. 1995; Laine et al. 2002; Erwin 2004).

The results show that Fourier analysis well separates bar/oval/lens structures from spiral arms, with oval/lens structures affecting both double-gaussian and multicomponent profiles. One of the interesting results from Table 4 is the large fraction of the sample galaxies that have a bar which is not associated with a strong extended oval. Cases like NGC 1387, NGC 1440, and NGC 3941 present some of the purest bars (in Fourier space at least) known. Nevertheless, our 2D decompositions give somewhat different views of the structures seen in some of these galaxies. For example, LSB05 and Laurikainen et al. (2006) note that including a lens in the decomposition models of NGC 1387, 1440, 1533, 1574, and 3941 improves the fits (but has no effect on bulge-to-total luminosity ratios); all of these show single gaussian Fourier profiles. In some of these cases, the lens in question is more of an outer lens (Kormendy 1979) than an inner lens and the features have little impact on the Fourier terms because they are not very barlike. In the cases of NGC 1533 and 1574, significant $m=2$ amplitude is seen outside the bar region (Figure 4). Fourier decomposition and 2D multi-component decompositions do not always detect the same structural components because the former is designed to characterize non-axisymmetric components, while the latter must model all components, both axisymmetric and nonaxisymmetric. The two approaches are complimentary, and we use Fourier analysis here only to characterize aspects of the bars seen.

In contrast to NGC 1387, 1440, and 3941, many galaxies have bars imbedded in more significant extended ovals. These ovals are often aligned with the main bar, but misalignment is also found. Also, the ovals may lie so far out that they are distinct entities in their own right. NGC 2859 is an example of an ansae-type bar imbedded in a considerably extended, aligned oval, while NGC 1079 is an example of an ansae-type bar in a slightly misaligned extended oval. It is important to note that the extended ovals are distinct from their associated bars. As seen in NGC 2859, the main bar has significant higherorder Fourier terms, while the extended oval only appears in the $m=2$ term.

The mean Fourier parameters for the objects fitted with single or double gaussians in Table 3 are summarized in Table 5. The results show that bars fitted by single gaussians on average are shorter than those requiring double gaussians and have a smaller gaussian width. The single gaussian bars have $\left\langle r_{2}\right\rangle=1.8 \pm 0.3 \mathrm{kpc}$ and $\left\langle\sigma_{2}\right\rangle=0.58 \pm 0.10 \mathrm{kpc}$, compared to $\left\langle r_{2}\right\rangle$ $=\left\langle r_{12}\right\rangle=2.8 \pm 0.4 \mathrm{kpc}$ and $\left\langle\sigma_{2}\right\rangle=\left\langle\sigma_{12}\right\rangle=$ $0.88 \pm 0.12 \mathrm{kpc}$ for the double gaussian bars. The mean absolute blue magnitudes are nevertheless very similar 
between the two groups (last line of Table 5). Single gaussian bars have smaller relative Fourier amplitudes of higher order terms, and less increase in $r_{m}$ with increasing $m$, as compared to at least the first gaussian component of the double gaussian bars. The gaussian widths tend to be larger for $m=2$ than for $m>2$.

\subsection{Elmegreen Bar Classifications}

An important additional piece of information is the classification of these galaxies according to their bar surface brightness profiles. Elmegreen and Elmegreen (1985) showed that bars can be divided into flat and exponential types according to the shapes of these profiles. Figure 7 shows surface brightness profiles along the primary bar major and minor axes for 25 of our sample galaxies. (The profiles shown for NGC 1317 are instead for the secondary bar.) The profiles are normalized to the radius $r_{2}$ (or the maximum of $r_{12}$ and $r_{22}$ ) of the $m=2$ Fourier term. Also, the surface brightness is shown relative to that at the radius $r_{2}$. From these profiles we have derived the classification of the main bar given in Column 5 of Table 4 . The profiles show a wide range of shapes that includes both of the Elmegreen types, although half of the galaxies are clearly intermediate between flat and exponential. Only two of our sample galaxies are of the exponential type, which is not unexpected given that Elmegreen and Elmegreen (1985) found exponential profiles mainly for later type galaxies. Table 4 also shows that there is no correlation between Elmegreen bar type and the Fourier profile characteristics. However, the ansae-type bars are more often of the flat type than are the regular bars.

\subsection{Bar Strengths}

Bar strengths provide an additional way of comparing the various Fourier profile categories among our sample galaxies. We have used the gravitational torque approach (Sanders \& Tubbs 1980; Combes \& Sanders 1981; Buta $\&$ Block 2001) to derive the bar strength, $Q_{b}$, from our gaussian-fitted or symmetry-mapped bar images. This parameter is derived assuming a constant mass-to-light ratio and negligible dark matter, but allows for the less flattened shape of the bulge and an exponential vertical density distribution having vertical scaleheight derived from a fraction of the radial scale length. The procedure is described by Laurikainen \& Salo (2002), Salo et al. (1999, 2004), and Laurikainen, Salo \& Buta (2004). From the force in the plane, we derive $Q_{b}$ as the maximum of the function

$$
Q_{T}(r)=\frac{\left|F_{T}(r, \phi)\right|_{\max }}{<\left|F_{R}(r, \phi)\right|>},
$$

where $\left|F_{T}(r, \phi)\right|_{\max }$ is the maximum tangential force and $\left\langle\left|F_{R}(r, \phi)\right|>\right.$ is the azimuthally-averaged radial force. The resulting values of $Q_{b}$ are compiled in Table 6 , and differ only slightly from the total nonaxisymmetric strength $Q_{g}$ presented for these galaxies by LSB05 and Laurikainen et al. (2006).

A comparison between Tables 4 and 6 shows that single gaussian bars have a lower average $Q_{b}$ than do double gaussian bars. For the 7 single gaussian cases, $\left\langle Q_{b}\right\rangle=$ $0.116 \pm 0.049$ (stan. dev.) while for 10 double gaussian cases, $\left\langle Q_{b}\right\rangle=0.222 \pm 0.106$ (stan. dev.). For the 9 remaining cases, $\left\langle Q_{b}\right\rangle=0.161 \pm 0.068$ (stan. dev.) using the main bar feature when multiple components are listed. The results suggest that single gaussian bars are weaker on average than double gaussian bars, although the two groups overlap somewhat in $Q_{b}$.

The " $Q_{b}$ family" in Table 6 is a quantitative family estimate based on $Q_{b}$ (Buta et al. 2005). The $Q_{b}$ family is SA if $Q_{b}<0.05, \mathrm{SAB}$ if $Q_{b}=0.05-0.10, \mathrm{SAB}$ if $Q_{b}=0.10-$ $0.20, \mathrm{SA} \underline{\mathrm{B}}$ if $Q_{b}=0.20-0.25$, and SB if $Q_{b} \geq 0.25$. These ranges were chosen by Buta et al. (2005) to approximate the way these classifications are often made, especially for the underline types. Interestingly, from Table 2, 17 of our galaxies are visually judged to be type SB, while only 6 are SB from the $Q_{b}$ family. The bars in these early-type galaxies are not as strong as they look owing to the significant background bulge and disk components in most cases. The strongest bar in our sample is that seen in NGC 1452, with $Q_{b}=0.42$. Although this kind of bar strength is not unusual for a late-type spiral, it is exceptional in our early-type galaxy sample.

\subsection{Evaluation of the Symmetry Assumption}

The symmetry assumption of relative Fourier intensity profiles was used by Buta, Block, \& Knapen (2003) as a straightforward way of separating bars from spirals that allows the determination of quantitative bar strength with the effects of spiral arm torques largely removed. The assumption was based on the appearance of such profiles in the $B$-band for six early-type barred galaxies (NGC 1398, 2217, 4440, 4643, 4650, and 4665; types S0 ${ }^{+}$ to Sab) shown by Ohta, Hamabe, \& Wakamatsu (1990). Although first suggested by $B$-band imaging, the nearIR is still a better band for evaluating this issue because of its reduced sensitivity to dust and star formation.

We have shown that relative Fourier intensity profiles of bars in early-type galaxies show considerable symmetry in some cases, and asymmetry in others. Here we evaluate the impact on $Q_{b}$ of applying the symmetry assumption to two early-type bars with asymmetric $I_{m} / I_{0}$ profiles.

In our sample, NGC 1452 is a strong case of asymmetry in its bar profile. Figure 8 shows two different applications of the symmetry assumption to the low order Fourier terms. In the first, the $m=2$ amplitudes are reflected around $r_{2}=24^{\prime \prime}$ while in the second these amplitudes are reflected around $r_{2}=30^{\prime \prime}$. The higher order terms simply follow these mappings with $r_{m}$ increasing with $m$. Figure 9] shows the light remaining after these bar extrapolations are applied. For the $r_{2}=24^{\prime \prime}$ case (left panel), the bar is clearly not fully removed, showing residual ansae. The $r_{2}=30^{\prime \prime}$ case does a much better removal of the bar. Still, one can see in the map (at the bar ends) where too much bar light has been removed, as expected. The $r_{2}=30^{\prime \prime}$ mapping is very similar to what we have used here for NGC 1512. The doublehumped representation in that case removes the observed bar from the $K_{s}$-band image very well, and reveals the spiral arms near the ends of the bar.

To evaluate the impact of the two representations of NGC 1452's bar on bar strength, we have computed $Q_{b}$ for the double-gaussian representation in Figure 4 and the two representations in Figure 8 In all three cases,

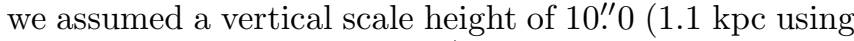
the distance from Tully 1988). The double-gaussian fit 
gives $Q_{b}=0.416$ (Table 6 ), while the $r_{2}=24^{\prime \prime}$ case gives $Q_{b}=0.356$ ( $14 \%$ difference) and the $r_{2}=30^{\prime \prime}$ case gives $Q_{b}=0.432$ ( $4 \%$ difference). Thus, even for an extreme case like NGC 1452, the uncertainty in $Q_{b}$ introduced by the symmetry assumption is relatively small. In most bar-spiral separations, residuals like those seen in Figure 9 do not occur (Buta et al. 2005).

Next we apply the symmetry assumption to NGC 4245, a case where the main peak in the bar might be lost if a strong spiral surrounded the bar. Figure [10] shows the rising portions of the $m=2,4$, and 6 profiles reflected around $r=33^{\prime \prime}$. This mapping removes most of the bar but leaves two weak enhancements near the bar ends (not shown). The double gaussian mapping in Figure 4 gives $Q_{b}=0.180$ (Table 6) while the Figure 10 mapping gives $Q_{b}=0.174$, a $3 \%$ difference.

These two cases demonstrate the effectiveness of the symmetry assumption as a way to separate spiral torques from bars and derive true bar strengths, not only for the majority of galaxies with well-define, symmetric Fourier profiles, but even for those where the Fourier profiles are less well-behaved, such as NGC 1452 and 4245.

\section{DISCUSSION}

Interpretation of the results in this paper requires a theory that accounts not only for bar strength and pattern speed, but also the varied shapes of bars. Studies by Athanassoula (2005 and references therein) show that angular momentum exchange is at the heart of all of these issues. Critical to the properties of bars is how much angular momentum is transferred to the halo. The effect depends on the density of matter in halo resonances and on how cold or hot the resonant material is. In order to absorb angular momentum a halo must be "live", as opposed to a rigid halo that cannot interact with other galaxy components. Cold, live halos can absorb so much angular momentum that a bar can grow very strong. Weaker bars form in warmer, smaller halos or rigid halos, while in hot disks, mainly ovals will form. The extreme effects of a live halo interaction has led to the possibility that bars might be found in systems lacking a background disk in the bar region. Gadotti and de Souza (2003) present two possible cases of this, although LSB05 present contrary evidence that does not support their claim.

Athanassoula computes relative Fourier mass profiles for her models that can be compared to the $I_{m} / I_{0}$ profiles presented in this paper. Provided the mass-to-light ratio in the $K_{s}$ band is relatively constant, such a comparison should be fair. The problems of comparing $n$-body models with real bars are summarized by Athanassoula \& Misiriotis (2002), who discuss the types of bars that develop in massive halo (MH), massive disk (MD), and intermediate models. Considering that the models are not of any specific galaxy, and are only a few of a large number of models actually computed, the relative Fourier mass profiles well resemble the observed profiles for some of our galaxies. The MH models show strong $m=2,4,6$, and 8 components while these are much weaker for the MD models, with $m=6$ and 8 being in the noise. The $\mathrm{MH}$ profiles most resemble those for NGC 1452, 2983, 4608 , and 4643 in our sample. The model bars have a fairly sharp outer edge, as is seen in these galaxies.

Athanassoula (2003) shows how the mass of the halo impacts the Fourier terms. More massive halos lead to stronger bars. Her most massive halo model (Mr3) has Fourier profiles similar to those of NGC 1452 and 4643, two of the strongest bars in our sample. Model MH1, with a less massive halo, has profiles similar to NGC 936 and 2787, while model MH2, with the lowest halo mass in the three illustrated, shows mainly an oval bar and has profiles resembling those of NGC 1302 and 2781.

The good agreement between the models and the observations is further shown by the simulations of Athanassoula, Lambert, \& Dehnen (2005), who evaluate the effects of a central mass concentration (CMC) on the evolution of the bar models in the previous papers. They show that a CMC has an effect on the higher $m$ terms, weakening their importance and leading to more $m=2$-dominated bars. Before the introduction of a CMC in their massive halo ( $\mathrm{MH}$ ) models, the relative Fourier mass profiles (their Figure 4) strongly resemble those we observe for NGC 4643, which has the sharpest outer edge of all the bars in our sample. In this model, the mass ratios $A_{4} / A_{2}=0.69, A_{6} / A_{0}=0.47$, and $A_{8} / A_{0}=0.36$, are very comparable with the intensity ratios found using the first gaussian component of NGC 4643: $I_{4} / I_{2}=0.66$, $I_{6} / I_{2}=0.45$, and $I_{8} / I_{2}=0.29$, respectively (from Table 3 ). Comparable values are found for NGC 1452 and NGC 4608. In their $\mathrm{MH}$ models with a $\mathrm{CMC}$, the profiles are more symmetric and the ratios above are considerably reduced. This is as observed for most of the other galaxies in our sample whose bars are weaker. Massive disk (MD) models in this paper also show similar effects, but the bars are weaker than for the $\mathrm{MH}$ models and the profile asymmetries are less important.

Bureau \& Athanassoula (2005) present three $n$-body models designed for deducing diagnostics of edge-on bars. These models are similar to those used by Athanassoula \& Misiriotis (2002) and include a weak bar, an intermediate strength bar, and a strong bar, with each model bar surrounded by a strong (and largely circular) stellar inner ring. The Fourier decompositions of these barred galaxy models again resemble the galaxies described above. In the strong bar model, the outer end of the bar has a sharp edge, similar to what is seen in NGC 1452 and 4643 in our sample. The strongest circular inner rings in our sample are seen in NGC 1452 and 4608 (Figure 2). Weaker circular inner rings are seen in NGC 936, 1317, and 2787, but in several of our galaxies the rings are highly elongated. Early-type barred galaxies have a wide range in intrinsic inner ring shapes, as is typical of such features (Buta 1995).

The relative Fourier intensity profiles of the bars in our sample could also include some of the effects of bar destruction. The bars in our sample that include overlapping extended ovals could be cases where the bar destruction was in progress at the time the galaxies became relatively deficient in gas. Bournaud \& Combes (2002) suggested that bar destruction in the absence of external gas accretion can lead to an oval lens feature (see also Kormendy 1979). An extreme example of this in our sample could be NGC 2859, where the impact of the oval lens extends considerably beyond the ends of the bar. Our Fourier analysis is efficient for separating the lens from the bar in this case, based on the disappearance of the higher order Fourier modes in the lens. Such an extended oval is only weakly seen in NGC 1452, 4608, or 4643, as 
if these bars were at their peaks and had not begun to disintegrate. These same three galaxies also have little or no central oval or secondary bar.

\section{CONCLUSIONS}

We have described a new survey designed to study the near-IR properties of bars in early-type (S0-Sa) disk galaxies. The goal of the survey is to obtain a statistically useful database that is comparable in size to that of the OSU survey of bright spiral galaxies, for the principal purpose of deriving the distribution of bar strengths in S0-Sa galaxies. LSB05 have presented a multicomponent decomposition analysis of 24 galaxies in our sample obtained thus far. Adding in a number of galaxies observed with the ESO NTT (Laurikainen et al. 2006), we have examined in this paper the relative Fourier intensity profiles of the bars in a subset of 26 early-type galaxies, for which the multi-component decomposition analysis provided deprojected images.

The results show that a significant fraction $(17 / 26)$ of the bars in our sample have simple relative Fourier intensity profiles that can be described in terms of single or double gaussians. The single gaussian Fourier profile types represent the simplest types of bars. The presence of extended ovals, secondary bars and nuclear ovals, as well as intermediate types of features, complicates many of the observed profiles. There is an indication in our sample that single gaussian bars are weaker on average than double gaussian bars.

Although gaussians can represent the $I_{m} / I_{0}$ profiles of some bars, the exact physical significance of such representations is unclear at the moment. Nevertheless, numerical bar models reproduce the types of Fourier profiles we observe in our early-type galaxy sample. We have shown that the profiles of the strongest bars in our sample resemble those found for massive halo models of barred galaxies where the angular momentum exchange between a bar and a massive live halo can be very effective. The weaker bars in our sample may show the effects of CMCs or have hotter and smaller halos, or just have more massive disks, than the stronger bars. In any case, it is clear that $I_{m} / I_{0}$ profiles provide a fruitful way of comparing models and simulations, and that further such comparisons may clarify the relative importance of different effects.

We have shown that early-type galaxy bars support the symmetry assumption used in bar-spiral separation studies. Even in an extreme case of very asymmetric $I_{m} / I_{0}$ profiles, the use of the symmetry assumption will likely lead to less than a $10 \%$ uncertainty in the estimates of relative bar torque strength $Q_{b}$ (BBK03).

We thank E. Athanassoula and an anonymous referee for helpful comments on the manuscript. RB acknowledges the support of NSF Grants AST 02-05143 and AST 05-07140 to the University of Alabama. EL and HS acknowledge the support of the Academy of Finland and the Magnus Ehrnrooth Foundation. JHK acknowledges the support of the Leverhulme Trust, and DLB acknowledges the support of the Anglo-American Chairman's Fund.

\section{REFERENCES}

Aaronson, M., Huchra, J., Mould, J., Schechter, P. L., and Tully, R. B. 1982, ApJ, 258, 64

Athanassoula, E. 2003, MNRAS, 341, 1179
Athanassoula, E. 2005, Celestial Mechanics and Dynamical Astronomy, 91, 9

Athanassoula, E., Lambert, J. C., and Dehnen, W. 2005, MNRAS, 363, 496

Athanassoula, E. \& Misiriotis, A. 2002, MNRAS, 330, 35

Block, D. L., Bournaud, F., Combes, F., Puerari, I., \& Buta, R. 2002, A\&A, 394, L35

Bournaud, F. \& Combes, F., 2002 A\&A, 392, 83

Bureau, M. \& Athanassoula, E. 2005, ApJ, 626, 159

Buta, R. 1990, ApJ, 356, 87

Buta, R. 1995, ApJS, 96, 39

Buta, R., Block, D. L., \& Knapen, J. H. 2003, AJ, 126, 1148 (BBK03)

Buta, R., Corwin, H. G., and Odewahn, S. C. 2006, The De Vaucouleurs Atlas of Galaxies, Cambridge, Cambridge University Press

Buta, R., Laurikainen, E., \& Salo, H. 2004, AJ, 127, 279 (BLS04)

Buta, R. and Purcell, G. B. 1998, AJ, 115, 484

Buta, R., Vasylyev, S., Salo, H., and Laurikainen, E. 2005, AJ, 130, 506

Combes, F. \& Sanders, R. H. 1981, A\&A, 96, 164

de Vaucouleurs, G. 1959, Handbuch der Physik, 53, 275

de Vaucouleurs, G. 1963, ApJS, 8, 31

de Vaucouleurs, G. et al. 1991, Third Reference Catalog of Bright Galaxies (New York: Springer) (RC3)

Elmegreen, B. G. \& Elmegreen, D. M. 1985, ApJ, 288, 438

Erwin, P. 2004, A\&A, 415, 941

Erwin, P., Beltrán, J., Graham, A. W., \& Beckman, J. E. 2003, ApJ, 597, 929

Erwin, P. \& Sparke, L. S. 1999, ApJ, 521, L37

Erwin, P. \& Sparke, L. S. 2003, ApJS, 146, 299

Eskridge, P., Frogel, J. A., Pogge, R. W., et al. 2002, ApJS, 143, 73

Gadotti, D. A. and de Souza, R. E. 2003, ApJ, 583, 75

Hubble, E. 1926, ApJ, 64, 321

Hubble, E. 1936. The Realm of the Nebulae, New York, Dover Publications

Kormendy, J. 1979, ApJ, 227, 714

Kormendy, J. and Norman, C. A. 1979, ApJ, 233, 539

Laine, S., Shlosman, I., Knapen, J. H., \& Peletier, R. F. 2002, ApJ, 567, 97

Laurikainen, E., Salo, H., \& Buta, R. 2004a, ApJ, 607, 103 (LSB04)

Laurikainen, E., Salo, H., \& Buta, R. 2005, MNRAS, 362, 1319 (LSB05)

Laurikainen, E., Salo, H., Buta, R., \& Vasylyev, S. 2004b, MNRAS, 355, 1251

Laurikainen, E., et al. 2006, in preparation

Ohta, K., Hamabe, M., \& Wakamatsu, K. 1990, ApJ, 357,71

Persson, S. E., Murphy, D. C., Krzeminski, W., Roth, \& Rieke, M. J. 1998, AJ, 116, 2475

Press, W. H., Flannery, B. P., Teukolsky, S. A. 1986, Numerical Recipes. The Art of Scientific Computing, Cambridge, Cambridge University Press

Quillen, A. C., Frogel, J. A., \& González, R. A. 1994, ApJ, 437, 162

Sandage, A. R. 1961, The Hubble Atlas of Galaxies, Carnegie Inst. of Wash. Publ. No. 618

Sandage, A. \& Bedke, J. S. 1994, The Carnegie Atlas of Galaxies, Carnegie Inst. of Wash. Publ. No. 638

Sanders, R. H. \& Tubbs, A. D. 1980, ApJ, 235, 803 
TABLE 1

Mean Properties of NiRSos Sample

\begin{tabular}{lccccc}
\hline \hline \multicolumn{1}{c}{ Sample } & $\left\langle M_{B}^{o}\right\rangle$ & mean error & $\langle T\rangle$ (RC3) & mean error & $N$ \\
\hline full sample & -20.044 & 0.078 & -1.595 & 0.128 & 190 \\
SA galaxies & -20.114 & 0.151 & -1.530 & 0.165 & 60 \\
SAB galaxies & -20.129 & 0.175 & -0.846 & 0.272 & 37 \\
SB galaxies & -19.735 & 0.094 & -0.905 & 0.184 & 56 \\
& & & & & \\
\hline
\end{tabular}

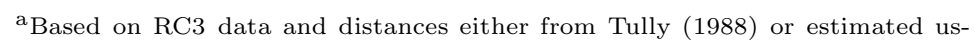
ing the linear Virgocentric flow model of Aaronson et al. (1982) and a Hubble constant of $75 \mathrm{~km} \mathrm{~s}^{-1} \mathrm{Mpc}^{-1}$.

TABLE 2

Fourier ANALYSIS SAMPLE ${ }^{\mathrm{a}}$

\begin{tabular}{|c|c|c|c|c|c|}
\hline $\begin{array}{c}\text { Galaxy } \\
1\end{array}$ & $\begin{array}{c}\text { Type } \\
2\end{array}$ & $\begin{array}{c}\log _{3} D_{o} \\
\quad\end{array}$ & $\begin{array}{c}M_{B}^{o} \\
4\end{array}$ & $\begin{array}{c}\mu_{o} \\
5\end{array}$ & $\begin{array}{c}\text { Telescope } \\
6\end{array}$ \\
\hline NGC 718 & $\left(\mathrm{R}^{\prime}\right) \mathrm{SAB}(\mathrm{rs}) \mathrm{a}$ & 1.38 & -19.4 & 31.65 & NOT \\
\hline NGC 936 & $\mathrm{SB}(\underline{\mathrm{rs}}) 0^{+}$ & 1.67 & -20.8 & 31.14 & NOT \\
\hline NGC 1022 & (R) $\overline{\mathrm{S}} \mathrm{B}(\underline{\mathrm{r} s}) \mathrm{a}$ pec & 1.38 & -19.4 & 31.33 & NOT \\
\hline NGC 1079 & $\left(\mathrm{R}_{1} \mathrm{R}_{2}^{\prime}\right) \overline{\mathrm{S}} \underline{\mathrm{A}} \mathrm{B}(\underline{\mathrm{r}} \mathrm{s}) \mathrm{a}$ & 1.53 & -19.0 & 31.13 & NTT \\
\hline NGC 1302 & $\left(\mathrm{R}_{2}^{\prime}\right) \mathrm{SAB}(\underline{\mathrm{r}} \mathrm{s}) \overline{0} / \mathrm{a}$ & 1.59 & -20.0 & 31.51 & NOT \\
\hline NGC 1317 & $\mathrm{SAB}(\mathrm{r}) \mathrm{a}$ & 1.44 & -19.3 & 31.14 & NTT \\
\hline NGC 1326 & $\left(\mathrm{R}_{1}\right) \mathrm{SAB}(\mathrm{r}) 0 / \mathrm{a}$ & 1.57 & -19.9 & 31.14 & NTT \\
\hline NGC 1387 & $\mathrm{SB}^{-}$ & 1.45 & -18.4 & 31.14 & NTT \\
\hline NGC 1440 & $\mathrm{SB}(\mathrm{s}) 0^{+}$ & 1.34 & -18.9 & 31.30 & NOT \\
\hline NGC 1452 & $\left(\mathrm{R}^{\prime}\right) \mathrm{SB}(\mathrm{r}) \mathrm{a}$ & 1.36 & -19.2 & 31.59 & NOT \\
\hline NGC 1512 & $\mathrm{SB}(\mathrm{r}) \mathrm{ab}$ & 1.95 & -18.9 & 29.90 & NTT \\
\hline NGC 1533 & $(\mathrm{RL}) \mathrm{SB}^{\circ}$ & 1.43 & -19.9 & 30.64 & NTT \\
\hline NGC 1574 & $\mathrm{SB}^{-}$ & 1.52 & -20.0 & 30.64 & NTT \\
\hline NGC 2217 & $(\mathrm{R}) \mathrm{SB}(\underline{\mathrm{r} s}) 0 / \mathrm{a}$ & 1.66 & -20.1 & 31.45 & NOT \\
\hline NGC 2273 & (RR)SÄB(rs)a & 1.54 & -20.3 & 32.26 & NOT \\
\hline NGC 2681 & (R)SAB $(\underline{\mathrm{rs}}) 0 / \mathrm{a}$ & 1.57 & -20.3 & 30.62 & NOT \\
\hline NGC 2781 & $\left(\mathrm{R}_{2}^{\prime}\right) \overline{\mathrm{SAB}} \overline{(\underline{\mathrm{r}} \mathrm{s})} 0 / \mathrm{a}$ & 1.45 & -19.9 & 32.20 & NOT \\
\hline NGC 2787 & $\mathrm{SB}(\mathrm{r}) 0^{+}$ & 1.48 & -19.2 & 30.58 & NOT \\
\hline NGC 2859 & (R) $\mathrm{SB}(\mathrm{rl}) 0^{+}$ & 1.62 & -20.4 & 32.03 & NOT \\
\hline NGC 2983 & (L)SB(s) $0^{+}$ & 1.38 & -19.6 & 32.19 & NOT \\
\hline NGC 3081 & $\left(\mathrm{R}_{1} \mathrm{R}_{2}^{\prime}\right) \mathrm{SAB}(\mathrm{r}) 0 / \mathrm{a}$ & 1.33 & -20.0 & 32.56 & NTT \\
\hline NGC 3941 & $\mathrm{SB}(\mathrm{s}) 0^{\circ}$ & 1.52 & -20.2 & 31.38 & NOT \\
\hline NGC 4245 & $\mathrm{SB}(\mathrm{r}) 0 / \mathrm{a}$ & 1.46 & -17.9 & 29.93 & NOT \\
\hline NGC 4596 & $\mathrm{SB}(\mathrm{rs}) 0 / \mathrm{a}$ & 1.58 & -19.6 & 31.13 & NOT \\
\hline NGC 4608 & $\mathrm{SB}(\mathrm{r}) 0 / \mathrm{a}$ & 1.49 & -19.2 & 31.13 & NOT \\
\hline NGC 4643 & $\mathrm{SB}(\mathrm{rl}) 0 / \mathrm{a}$ & 1.49 & -20.6 & 32.05 & NOT \\
\hline
\end{tabular}

${ }^{a}$ Explanation of columns: (1) galaxy name; (2) new $B$-band morphological classification from the de Vaucouleurs Atlas of Galaxies (Buta, Corwin, and Odewahn 2006), except for NGC 1440,2781, 2787, and 3941 whose types are in the same system but based on inspection of photographic images in Sandage and Bedke (1994); (3) logarithm of isophotal diameter at $\mu_{B}=$ $25.00 \mathrm{mag} \operatorname{arcsec}^{-2}$, corrected for extinction and inclination (from RC3); (4) absolute $B$-band magnitude based on RC3 data and (5) the distance modulus from Tully (1988), which is based on a Hubble constant of $75 \mathrm{~km}$ $\mathrm{s}^{-1} \mathrm{Mpc}^{-1}$ in conjunction with a linear Virgocentric flow model (Aaronson et al. 1982); (6) telescope used for near-IR imaging: NOT = Nordic Optical 2.5-m Telescope; NTT = ESO 3.5-m New Technology Telescope.

Tully, R. B. 1988, Nearby Galaxies Catalogue, Cambridge, Cambridge University Press

Wozniak, H., Friedli, D., Martinet, L., Martin, P., \& Bratschi, P. 1995, A\&AS, 111, 115 
TABLE 3

Gaussian Fourier Components for 24 Early-Type Barred and Oval Galaxies ${ }^{\mathrm{a}}$

\begin{tabular}{|c|c|c|c|c|c|c|c|c|c|c|c|c|c|}
\hline $\begin{array}{c}\text { Galaxy } \\
1\end{array}$ & $\begin{array}{c}A_{2} \\
2\end{array}$ & $\begin{array}{c}A_{4} \\
3\end{array}$ & $\begin{array}{c}A_{6} \\
4\end{array}$ & $\begin{array}{c}A_{8} \\
5\end{array}$ & $\begin{array}{c}r_{2} \\
6\end{array}$ & $\begin{array}{c}r_{4} \\
7\end{array}$ & $\begin{array}{c}r_{6} \\
8\end{array}$ & $\begin{array}{c}r_{8} \\
9\end{array}$ & $\begin{array}{l}\sigma_{2} \\
10\end{array}$ & $\begin{array}{l}\sigma_{4} \\
11\end{array}$ & $\begin{array}{l}\sigma_{6} \\
12\end{array}$ & $\begin{array}{l}\sigma_{8} \\
13\end{array}$ & $\begin{array}{c}r_{2} / r_{o} \\
14\end{array}$ \\
\hline N0718-1 & 0.35 & 0.12 & 0.05 & 0.00 & 13.1 & 12.6 & 13.1 & 0.0 & 4.5 & 2.4 & 2.1 & 0.0 & 0.18 \\
\hline N0718-2 & 0.40 & 0.18 & 0.09 & 0.00 & 22.8 & 20.1 & 21.1 & 0.0 & 3.7 & 2.7 & 2.4 & 0.0 & 0.32 \\
\hline N0936-1 & 0.34 & 0.29 & 0.17 & 0.09 & 27.3 & 36.9 & 37.5 & 38.5 & 8.8 & 9.4 & 8.4 & 7.3 & 0.19 \\
\hline N0936-2 & 0.43 & 0.00 & 0.00 & 0.00 & 42.5 & 0.0 & 0.0 & 0.0 & 10.0 & 0.0 & 0.0 & 0.0 & 0.30 \\
\hline N1079 & 0.68 & 0.28 & 0.13 & 0.06 & 42.4 & 40.2 & 39.9 & 39.9 & 14.7 & 8.8 & 6.3 & 4.7 & 0.42 \\
\hline N1302-1 & 0.30 & 0.09 & 0.00 & 0.00 & 18.3 & 20.0 & 0.0 & 0.0 & 5.3 & 4.7 & 0.0 & 0.0 & 0.16 \\
\hline N1302-2 & 0.23 & 0.06 & 0.00 & 0.00 & 30.3 & 27.4 & 0.0 & 0.0 & 5.3 & 2.2 & 0.0 & 0.0 & 0.26 \\
\hline N1317 & 0.36 & 0.18 & 0.11 & 0.06 & 4.7 & 5.1 & 5.2 & 5.3 & 2.1 & 1.6 & 1.3 & 1.3 & 0.06 \\
\hline N1326-1 & 0.08 & 0.09 & 0.03 & 0.04 & 20.1 & 26.5 & 29.9 & 28.2 & 4.1 & 5.6 & 3.3 & 5.5 & 0.18 \\
\hline N1326-2 & 0.64 & 0.22 & 0.09 & 0.03 & 41.9 & 41.5 & 37.1 & 39.0 & 17.3 & 11.6 & 10.8 & 6.4 & 0.38 \\
\hline N1387 & 0.39 & 0.15 & 0.06 & 0.03 & 18.3 & 17.9 & 17.2 & 17.0 & 6.0 & 5.1 & 4.9 & 4.9 & 0.22 \\
\hline N1440 & 0.48 & 0.23 & 0.11 & 0.05 & 17.3 & 18.4 & 19.4 & 19.6 & 6.8 & 4.7 & 3.7 & 4.4 & 0.26 \\
\hline N1452-1 & 0.75 & 0.53 & 0.43 & 0.28 & 20.5 & 26.8 & 31.0 & 31.4 & 7.3 & 7.8 & 8.4 & 7.7 & 0.30 \\
\hline N1452-2 & 0.67 & 0.36 & 0.18 & 0.12 & 38.3 & 41.9 & 43.7 & 41.6 & 10.0 & 7.0 & 4.3 & 7.1 & 0.56 \\
\hline N1533 & 0.42 & 0.17 & 0.07 & 0.03 & 19.1 & 20.1 & 21.7 & 22.2 & 7.6 & 5.9 & 5.9 & 6.2 & 0.24 \\
\hline N1574 & 0.32 & 0.14 & 0.06 & 0.03 & 13.3 & 13.5 & 13.5 & 13.5 & 4.3 & 3.6 & 3.1 & 3.7 & 0.13 \\
\hline $\mathrm{N} 2217$ & 0.51 & 0.25 & 0.15 & 0.09 & 33.4 & 32.9 & 34.0 & 34.5 & 11.7 & 9.4 & 7.4 & 7.5 & 0.24 \\
\hline N2273-1 & 0.20 & 0.23 & 0.15 & 0.08 & 15.0 & 18.4 & 19.1 & 19.5 & 3.2 & 4.8 & 3.9 & 3.7 & 0.14 \\
\hline N2273-2 & 0.60 & 0.20 & 0.06 & 0.05 & 30.9 & 36.0 & 39.3 & 41.5 & 12.1 & 6.7 & 6.7 & 8.2 & 0.30 \\
\hline N2681 & 0.25 & 0.06 & 0.03 & 0.00 & 14.9 & 14.9 & 14.6 & 0.0 & 5.0 & 3.8 & 3.2 & 0.0 & 0.13 \\
\hline N2781-1 & 0.47 & 0.00 & 0.00 & 0.00 & 36.4 & 0.0 & 0.0 & 0.0 & 8.1 & 0.0 & 0.0 & 0.0 & 0.43 \\
\hline N2781-2 & 0.33 & 0.00 & 0.00 & 0.00 & 54.8 & 0.0 & 0.0 & 0.0 & 8.4 & 0.0 & 0.0 & 0.0 & 0.65 \\
\hline $\mathrm{N} 2787$ & 0.52 & 0.21 & 0.11 & 0.06 & 34.3 & 36.3 & 36.6 & 37.3 & 10.2 & 7.9 & 7.2 & 6.2 & 0.38 \\
\hline N2859-1 & 0.56 & 0.22 & 0.11 & 0.06 & 40.2 & 39.6 & 40.2 & 40.6 & 13.5 & 11.7 & 9.1 & 8.7 & 0.32 \\
\hline N2859-2 & 0.37 & 0.00 & 0.00 & 0.00 & 66.1 & 0.0 & 0.0 & 0.0 & 11.6 & 0.0 & 0.0 & 0.0 & 0.53 \\
\hline N2983-1 & 0.32 & 0.44 & 0.27 & 0.17 & 14.9 & 25.4 & 25.9 & 26.4 & 4.7 & 7.3 & 5.9 & 5.4 & 0.21 \\
\hline N2983-2 & 0.74 & 0.00 & 0.00 & 0.00 & 25.4 & 0.0 & 0.0 & 0.0 & 7.6 & 0.0 & 0.0 & 0.0 & 0.35 \\
\hline N3081 & 0.28 & 0.10 & 0.05 & 0.00 & 5.4 & 5.3 & 5.2 & 0.0 & 2.0 & 1.6 & 1.4 & 0.0 & 0.08 \\
\hline N3941 & 0.33 & 0.14 & 0.05 & 0.00 & 20.4 & 20.8 & 20.3 & 0.0 & 5.0 & 3.4 & 2.6 & 0.0 & 0.21 \\
\hline N4245-1 & 0.28 & 0.11 & 0.08 & 0.05 & 19.7 & 23.0 & 26.3 & 29.6 & 6.8 & 5.3 & 5.6 & 4.4 & 0.23 \\
\hline N4245-2 & 0.49 & 0.17 & 0.09 & 0.04 & 38.4 & 38.1 & 38.5 & 38.2 & 8.7 & 8.4 & 4.2 & 2.5 & 0.44 \\
\hline N4596-1 & 0.66 & 0.26 & 0.11 & 0.09 & 38.6 & 39.8 & 39.2 & 44.5 & 14.1 & 8.8 & 6.9 & 8.4 & 0.34 \\
\hline N4596-2 & 0.45 & 0.30 & 0.22 & 0.12 & 61.0 & 57.4 & 53.8 & 56.6 & 8.1 & 10.4 & 8.6 & 6.9 & 0.54 \\
\hline N4608-1 & 0.62 & 0.40 & 0.32 & 0.22 & 25.9 & 33.1 & 36.4 & 37.7 & 10.8 & 10.1 & 9.5 & 9.3 & 0.28 \\
\hline N4608-2 & 0.42 & 0.12 & 0.00 & 0.00 & 43.3 & 41.7 & 0.0 & 0.0 & 10.1 & 9.5 & 0.0 & 0.0 & 0.47 \\
\hline N4643-1 & 0.85 & 0.56 & 0.38 & 0.25 & 33.3 & 39.3 & 41.0 & 41.8 & 13.2 & 12.1 & 10.8 & 10.0 & 0.36 \\
\hline N4643-2 & 0.38 & 0.14 & 0.10 & 0.09 & 54.6 & 54.7 & 54.2 & 53.8 & 7.9 & 3.5 & 3.2 & 3.8 & 0.59 \\
\hline
\end{tabular}

a Explanations of columns: (1) Galaxy name. If a double gaussian was fitted to the $I_{m} / I_{0}$ profiles, the first gaussian is listed as "-1" while the second is "-2". (2-5): gaussian relative amplitudes $A_{m}$ (equation 1) for $m=2,4,6$, and 8. For a double gaussian fit (equation 2), $A_{1 m}$ is listed on the first line and $A_{2 m}$ is listed on the second line for a given galaxy. (6-9): mean radii $r_{m}$ (or $r_{1 m}$ and $r_{2 m}$ for a double gaussian fit), in arcseconds. (10-13): gaussian width $\sigma_{m}$ (or $\sigma_{1 m}$ and $\sigma_{2 m}$ for a double gaussian fit), in arcseconds. (14) ratio of radius $r_{2}$ to the radius $r_{o}=D_{o} / 2$ of the standard isophote having $\mu_{B}=25.00 \mathrm{mag}^{\operatorname{arcsec}}-2$. 
TABLE 4

Summary of Primary Components ${ }^{\mathrm{a}}$

\begin{tabular}{|c|c|c|c|c|}
\hline $\begin{array}{c}\text { Galaxy } \\
1\end{array}$ & $\begin{array}{l}\text { Fourier } \\
\text { profile type } \\
2\end{array}$ & $\begin{array}{c}\text { Primary } \\
\text { feature } \\
3\end{array}$ & $\begin{array}{c}\text { Other } \\
\text { features } \\
4\end{array}$ & $\begin{array}{l}\text { Main bar } \\
\text { profile type } \\
5\end{array}$ \\
\hline NGC 936 & DGFP & ansae bar & & flat \\
\hline NGC 1022 & MCFP & distorted bar & oval disk & intermediate \\
\hline NGC $1079^{\mathrm{b}}$ & MCFP & ansae bar & extended misaligned oval & flat \\
\hline NGC 1326 & MCFP & ansae bar & extended aligned oval & flat \\
\hline NGC 1387 & SGFP & regular bar & & exponential \\
\hline NGC 1440 & SGFP & regular bar & & intermediate \\
\hline NGC 1452 & DGFP & regular bar & & flat \\
\hline NGC 1512 & MCFP & ansae bar & extended aligned oval & flat \\
\hline NGC 2681 & MCFP & regular bar & extended misaligned ansae oval & intermediate \\
\hline NGC 2781 & DGFP & oval & extended aligned oval & intermediate \\
\hline NGC 2787 & SGFP & ansae bar & & flat \\
\hline NGC 2859 & DGFP & ansae bar & inner oval, extended aligned outer oval & flat \\
\hline NGC 2983 & DGFP & ansae bar & & flat \\
\hline NGC 3081 & MCFP & ring/oval & aligned intermediate bar & flat \\
\hline NGC 3941 & SGFP & ansae bar & & intermediate \\
\hline NGC 4245 & DGFP & regular bar & extended aligned oval & intermediate \\
\hline NGC 4596 & DGFP & ansae bar & extended aligned oval & intermediate \\
\hline NGC 4608 & DGFP & regular bar & & intermediate \\
\hline NGC 4643 & DGFP & regular bar & & intermediate \\
\hline
\end{tabular}

${ }^{a}$ Explanation of columns: (1) Galaxy name; (2) the Fourier $I_{m} / I_{0}$ profile type based on gaussian fitting, where $\mathrm{SGFP}=$ single gaussian Fourier profile, DGFP = double gaussian Fourier profile, and MCFP = multi-component Fourier profile (section 5.2); (3) morphology of primary bar or bar-like feature; (4) additional non-nuclear bar-like features; (5) Elmegreen and Elmegreen (1985) primary bar classification

${ }^{\mathrm{b}}$ Deprojection uncertainties complicated analysis; inner regions affected by bulge deprojection stretch. 
TABLE 5

Mean Fourier Parameters ${ }^{\mathrm{a}}$

\begin{tabular}{|c|c|c|c|c|c|c|c|c|}
\hline Parameter & mean & $\begin{array}{l}\text { standard } \\
\text { deviation }\end{array}$ & $\begin{array}{l}\text { mean } \\
\text { error }\end{array}$ & $\mathrm{n}$ & mean & $\begin{array}{l}\text { standard } \\
\text { deviation }\end{array}$ & $\begin{array}{l}\text { mean } \\
\text { error }\end{array}$ & $\mathrm{n}$ \\
\hline 1 & 2 & 3 & 4 & 5 & 6 & 7 & & 9 \\
\hline & & SGFP Cases & & & & DGFP Cases & & \\
\hline$<r_{2}>(\mathrm{kpc})$ & 1.76 & 0.74 & 0.28 & 7 & 2.68 & 1.31 & 0.41 & 10 \\
\hline$<s_{2}>(\mathrm{kpc})$ & 0.58 & 0.26 & 0.10 & 7 & 0.88 & 0.38 & 0.12 & 10 \\
\hline$<r_{22}>(\mathrm{kpc})$ & $\ldots$ & $\ldots$ & $\ldots$ & .. & 4.39 & 1.96 & 0.62 & 10 \\
\hline$<s_{22}>(\mathrm{kpc})$ & $\ldots$ & $\ldots$ & $\ldots$ & .. & 0.84 & 0.31 & 0.10 & 10 \\
\hline$<I_{4} / I_{2}>$ & 0.43 & 0.04 & 0.01 & 7 & 0.64 & 0.33 & 0.11 & 9 \\
\hline$<I_{6} / I_{2}>$ & 0.20 & 0.05 & 0.02 & 7 & 0.44 & 0.22 & 0.08 & 8 \\
\hline$<I_{8} / I_{2}>$ & 0.10 & 0.04 & 0.01 & 6 & 0.28 & 0.14 & 0.05 & 8 \\
\hline$<I_{10} / I_{2}>$ & 0.06 & 0.03 & 0.01 & 6 & 0.19 & 0.11 & 0.04 & 8 \\
\hline$<I_{42} / I_{22}>$ & $\ldots$ & $\ldots$. & $\ldots$ & .. & 0.41 & 0.16 & 0.07 & 6 \\
\hline$<I_{62} / I_{22}>$ & $\ldots$ & $\ldots$ & $\ldots$ & .. & 0.30 & 0.14 & 0.07 & 4 \\
\hline$<I_{82} / I_{22}>$ & $\ldots$ & $\ldots$ & $\ldots$ & .. & 0.19 & 0.08 & 0.04 & 4 \\
\hline$<I_{102} / I_{22}>$ & $\ldots$ & $\ldots$ & $\ldots$ & .. & 0.11 & 0.07 & 0.04 & 3 \\
\hline$<r_{4} / r_{2}>$ & 1.02 & 0.04 & 0.01 & 7 & 1.23 & 0.21 & 0.07 & 9 \\
\hline$<r_{6} / r_{2}>$ & 1.04 & 0.07 & 0.03 & 7 & 1.33 & 0.25 & 0.09 & 8 \\
\hline$<r_{8} / r_{2}>$ & 1.06 & 0.08 & 0.03 & 6 & 1.39 & 0.24 & 0.08 & 8 \\
\hline$<r_{10} / r_{2}>$ & 1.12 & 0.14 & 0.06 & 6 & 1.44 & 0.25 & 0.09 & 8 \\
\hline$<r_{42} / r_{22}>$ & $\ldots$ & $\ldots$ & $\ldots$ & .. & 0.98 & 0.06 & 0.03 & 6 \\
\hline$<r_{62} / r_{22}>$ & $\ldots$ & $\ldots$ & $\ldots$ & .. & 1.00 & 0.11 & 0.05 & 4 \\
\hline$<r_{82} / r_{22}>$ & $\ldots$ & $\ldots$ & $\ldots$ & .. & 1.00 & 0.07 & 0.03 & 4 \\
\hline$<r_{102} / r_{22}>$ & $\ldots$ & $\ldots$ & & & 1.04 & 0.11 & 0.07 & 3 \\
\hline$<s_{4} / s_{2}>$ & 0.77 & 0.07 & 0.03 & 7 & 0.96 & 0.26 & 0.09 & 9 \\
\hline$<s_{6} / s_{2}>$ & 0.68 & 0.11 & 0.04 & 7 & 0.88 & 0.24 & 0.09 & 8 \\
\hline$<s_{8} / s_{2}>$ & 0.74 & 0.12 & 0.05 & 6 & 0.81 & 0.20 & 0.07 & 8 \\
\hline$\left\langle s_{10} / s_{2}\right\rangle$ & 1.05 & 0.36 & 0.15 & 6 & 1.18 & 0.28 & 0.10 & 8 \\
\hline$<s_{42} / s_{22}>$ & $\ldots$. & $\ldots$ & $\ldots$ & .. & 0.79 & 0.33 & 0.14 & 6 \\
\hline$<s_{62} / s_{22}>$ & $\ldots$ & $\ldots$ & $\ldots$ & .. & 0.60 & 0.31 & 0.16 & 4 \\
\hline$<s_{82} / s_{22}>$ & $\ldots$ & $\ldots$ & $\ldots$ & .. & 0.58 & 0.25 & 0.12 & 4 \\
\hline$\left.<s_{102} / s_{22}\right\rangle$ & $\ldots$ & $\ldots$ & $\ldots$ & .. & 0.92 & 0.44 & 0.26 & 3 \\
\hline$<M_{B}^{o}>$ & -19.53 & 0.70 & 0.26 & 7 & -19.72 & 0.84 & 0.27 & 10 \\
\hline
\end{tabular}

a Explanation of columns: (1) Fourier parameter (linear diameters use the distance moduli from Table 1); (2)-(5) parameter means, standard deviations, mean errors, and the number of objects for SGFP cases from Table 4; (6)-(9) the same for DGFP cases from Table 4. 
TABLE 6

Bar Strengths and $Q_{b}$ Families $^{\mathrm{a}}$

\begin{tabular}{|c|c|c|c|}
\hline $\begin{array}{c}\text { Galaxy } \\
1\end{array}$ & $\begin{array}{c}Q_{b} \text { Family } \\
2\end{array}$ & $\begin{array}{c}Q_{b} \\
3\end{array}$ & $\begin{array}{c}\text { mean errol } \\
4\end{array}$ \\
\hline NGC 718 & SAB & 0.124 & 0.000 \\
\hline NGC 936 & $\mathrm{SAB}$ & 0.201 & 0.028 \\
\hline NGC 1022 & $\mathrm{SA} \overline{\mathrm{B}}$ & 0.142 & 0.016 \\
\hline NGC 1079 & $\mathrm{SAB}$ & 0.242 & 0.052 \\
\hline NGC 1302 & $\mathrm{SA} \overline{\mathrm{B}}$ & 0.130 & 0.007 \\
\hline NGC 1317-primary bar & $\mathrm{SAB}$ & 0.091 & 0.008 \\
\hline NGC 1317 -secondary bar & $\mathrm{SAB}$ & 0.086 & 0.006 \\
\hline NGC 1326 & $\mathrm{SAB}$ & 0.161 & 0.014 \\
\hline NGC 1387 & SABB & 0.065 & 0.002 \\
\hline NGC 1440 & $\mathrm{SAB}$ & 0.141 & 0.004 \\
\hline NGC 1452 & SB & 0.416 & 0.035 \\
\hline NGC 1512 & SB & 0.270 & 0.015 \\
\hline NGC 1533 & SAB & 0.107 & 0.002 \\
\hline NGC 1574 & SAB & 0.064 & 0.006 \\
\hline NGC 2217 & $\mathrm{SAB}$ & 0.170 & 0.008 \\
\hline NGC 2273 & $\mathrm{SA} \underline{B}$ & 0.209 & 0.001 \\
\hline NGC 2681-inner bar & $\mathrm{SA}^{-}$ & 0.039 & 0.003 \\
\hline NGC 2681-outer oval & SAB & 0.061 & 0.004 \\
\hline NGC 2781 & $\mathrm{SAB}$ & 0.066 & 0.001 \\
\hline NGC 2787 & $\mathrm{SAB}$ & 0.182 & 0.051 \\
\hline NGC 2859 & $\mathrm{SAB}$ & 0.105 & 0.004 \\
\hline NGC 2983 & SB & 0.297 & 0.007 \\
\hline NGC 3081-primary bar & SAB & 0.069 & 0.001 \\
\hline NGC 3081-primary oval & $\mathrm{S} \overline{\mathrm{A}} \mathrm{B}$ & 0.153 & 0.013 \\
\hline NGC 3081-secondary bar & $\mathrm{SAB}$ & 0.066 & 0.004 \\
\hline NGC 3941 & $\mathrm{SAB}$ & 0.085 & 0.005 \\
\hline NGC 4245 & $\mathrm{SAB}$ & 0.180 & 0.002 \\
\hline NGC 4596 & SB & 0.271 & 0.061 \\
\hline NGC 4608 & SB & 0.252 & 0.005 \\
\hline NGC 4643 & SB & 0.299 & 0.002 \\
\hline
\end{tabular}

${ }^{a}$ Explanation of columns: (1) Galaxy name; (2) quantitative family estimate following Buta et al. (2005); (3) maximum relative bar torque based on gaussian and other mappings; (4) mean error excluding systematic effects.

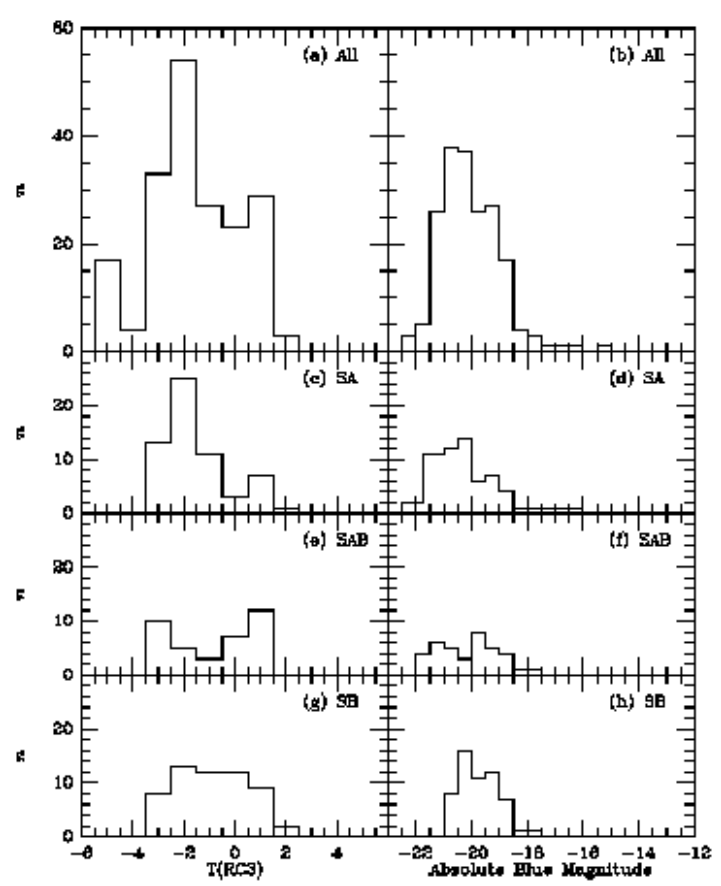

FIG. 1.-

FIG. 1.- Histograms of numbers of NIRSOS galaxies versus RC3 type index $T$ and absolute blue magnitude $M_{B}^{o}$ based on RC3 data and distances (mainly from Tully 1988) using a Hubble constant of $75 \mathrm{~km} \mathrm{~s}^{-1} \mathrm{Mpc}^{-1}$. The top two graphs are for the full sample, while the lower ones are divided according to RC3 family.

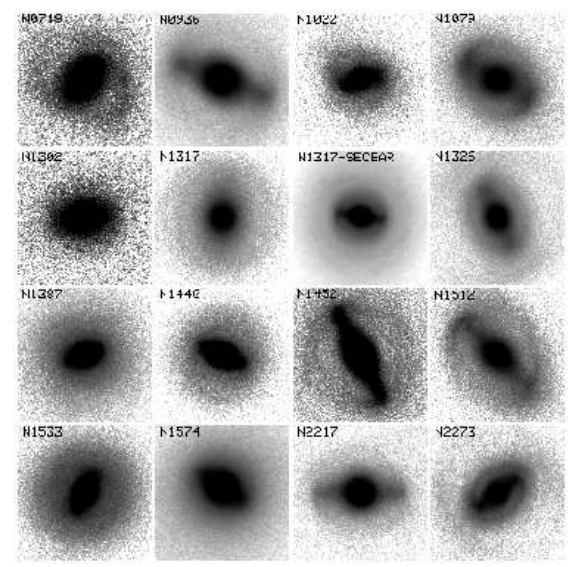

FIG. 2.- 


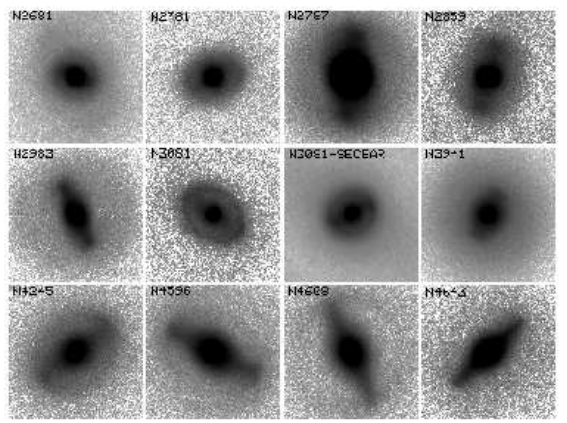

FIG. 2 (CONT.).- Deprojected $K_{s}$-band images of 26 NIRS0S galaxies. These are displayed mostly to emphasize the primary bars and outer disks, but not necessarily any nuclear structure. The exceptions are the secondary bars of NGC 1317 and 3081 , indicated by "SECBAR." The dimensions of each square are 1'96 $\times 1$ '.96 except for the following: NGC 1079, 1317, 1326, 1533, 3081 $\left(2^{\prime} .47 \times 22^{\prime} .47\right) ;$ NGC $2781,2859,4596,4643\left(2{ }^{\prime} .73 \times 2{ }^{\prime} .73\right) ;$ NGC $1512\left(3.44 \times 33^{\prime} 44\right)$; and the secondary bar closeups of NGC 1317, $3081\left(0^{\prime} 62 \times 00^{\prime} 62\right)$.
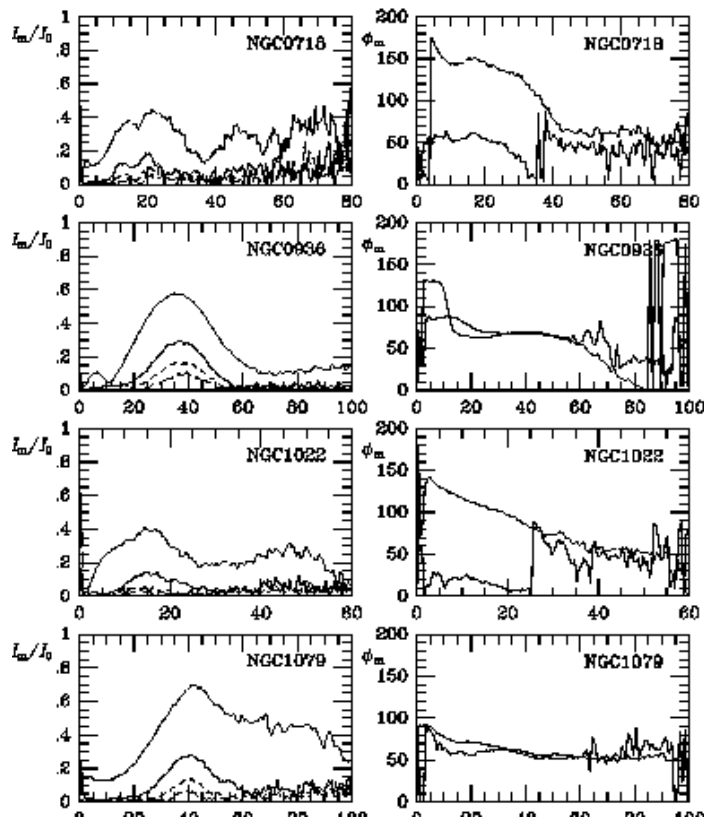

FIG. 3.-
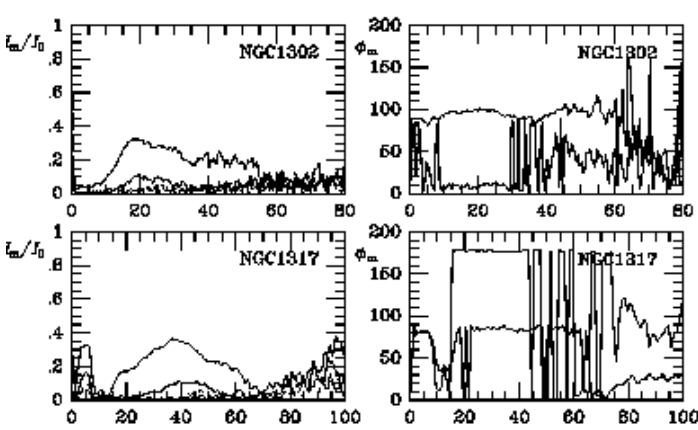

${ }_{\phi_{\mathrm{m}}}^{200} \mathrm{E}^{1}$
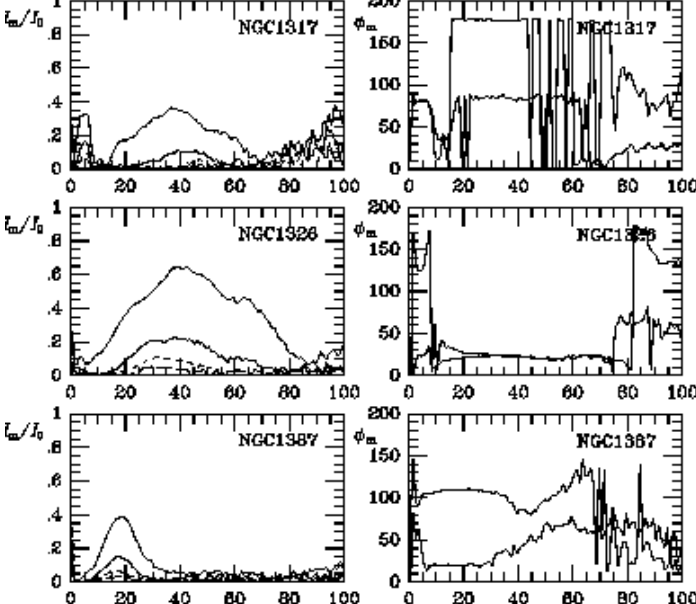

FIG. 3 (CONT.).-
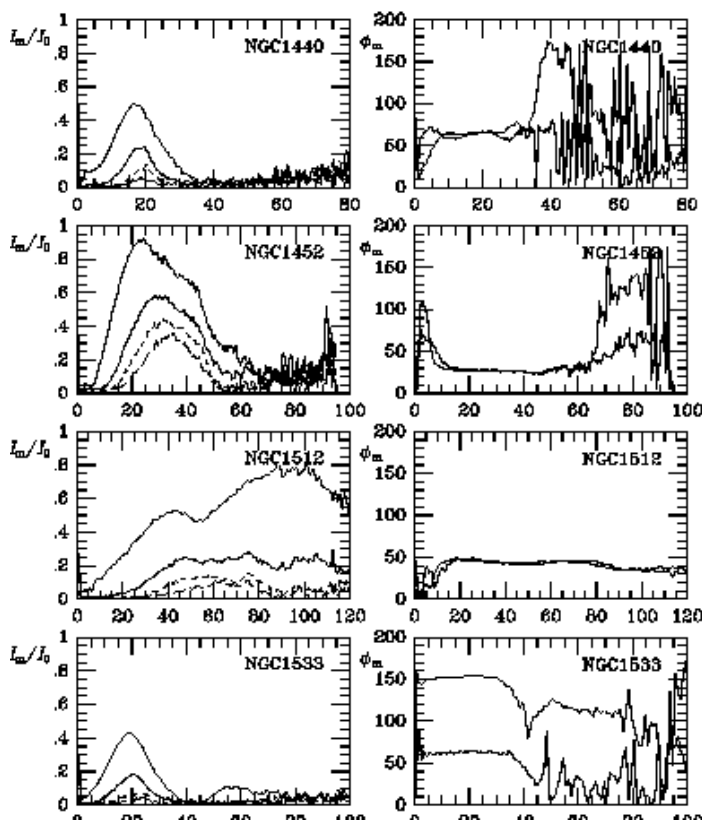

Fig. 3 (CONT.).- 

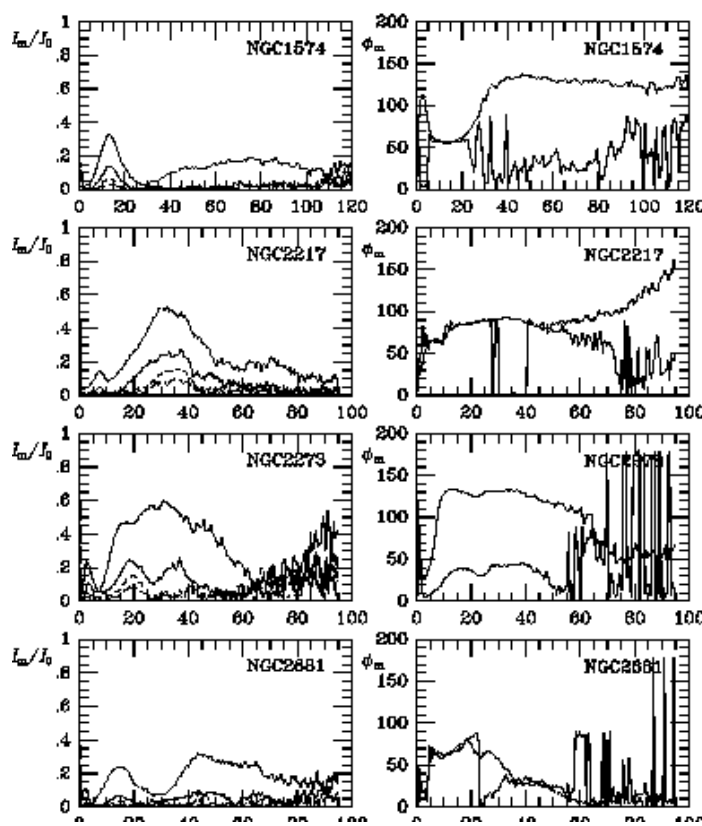

Fig. 3 (CONT.).-
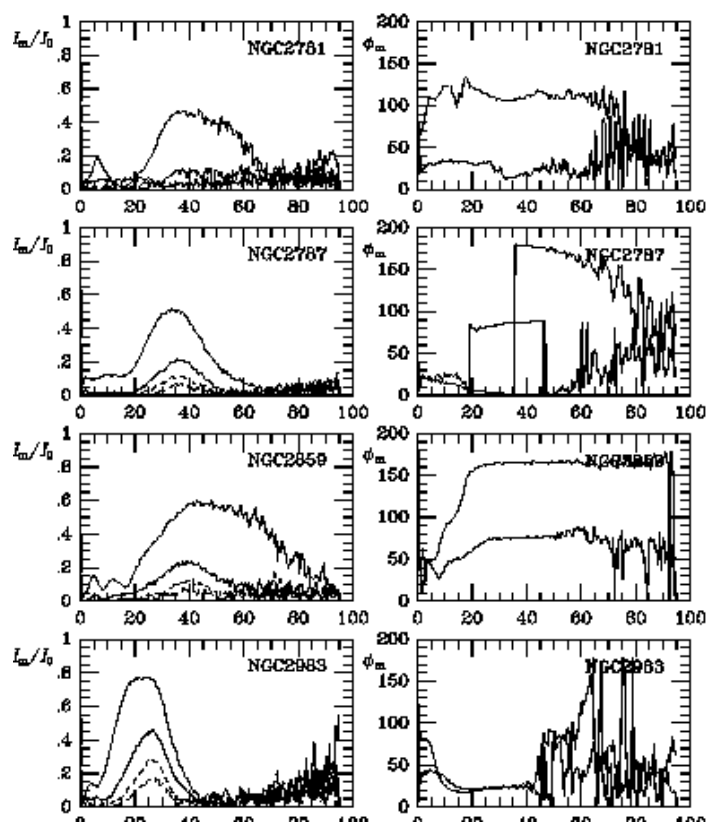

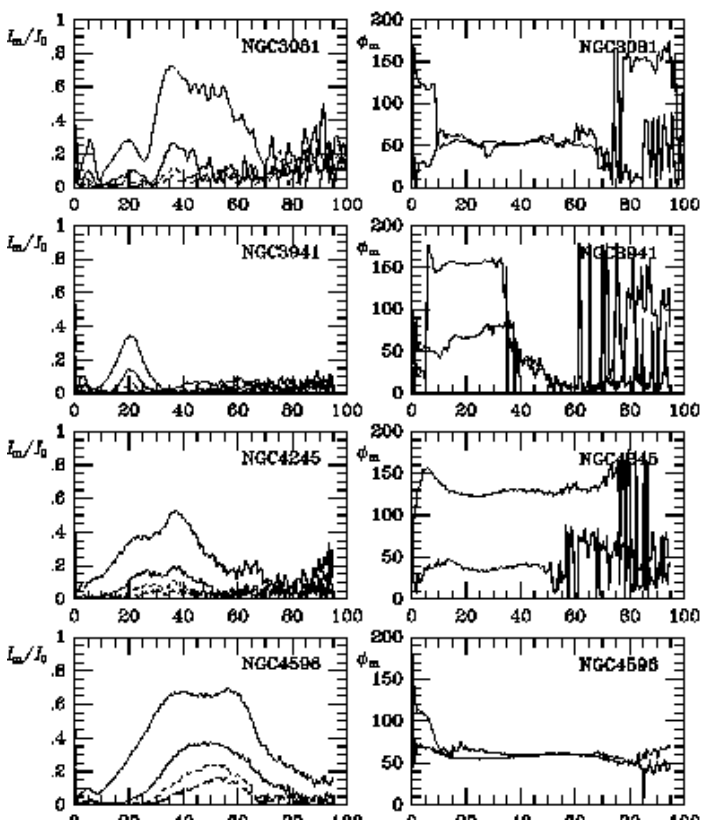

Fig. 3 (CONT.).-

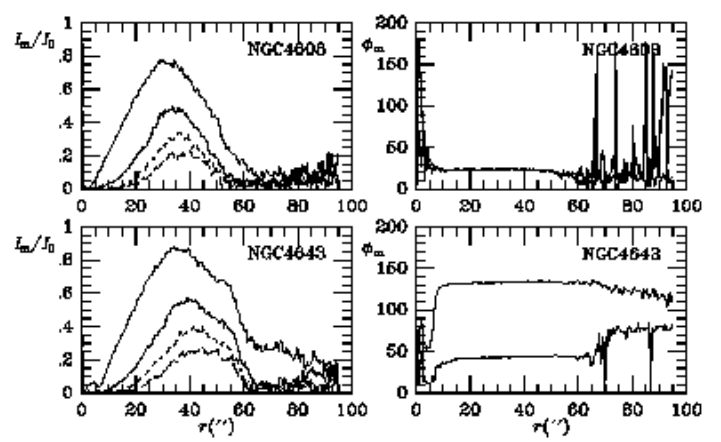

FIG. 3 (CONT.).- Relative Fourier intensity and phase profiles for 26 early-type disk galaxies. The left panels show the $I_{m} / I_{0}$ profiles for $m=2$ (solid curves), 4 (dotted curves), 6 (dashed curves), and 8 (dash-dot curves). The right panels show the phases $\phi_{m}$ for $m=2$ (solid curves) and 4 (dotted curves). The $360^{\circ} / m$ periodicity causes the sharp changes in phase in many of the plots.

FIG. 3 (CONT.).- 

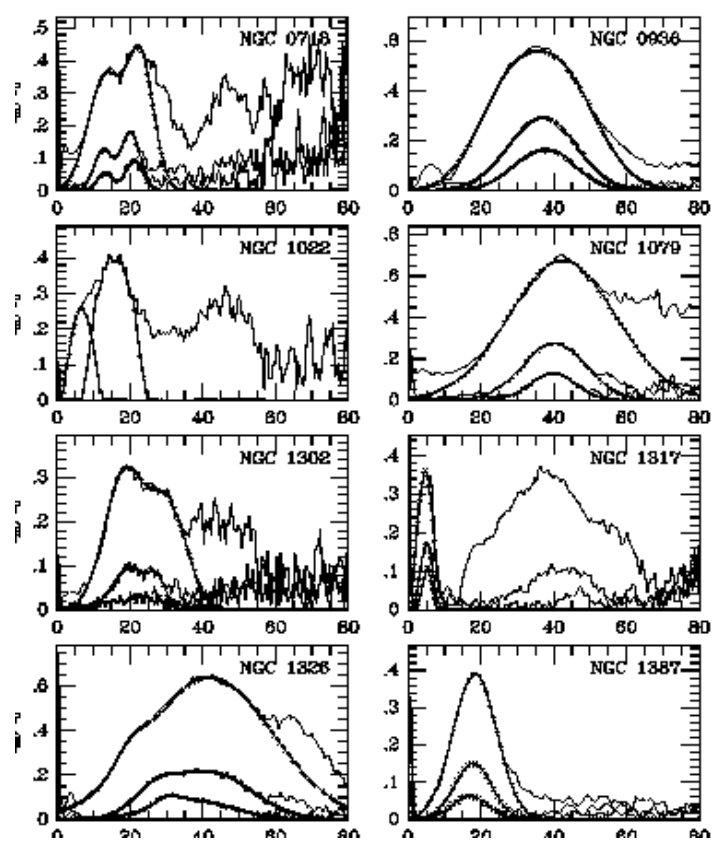

FIG. 4.-
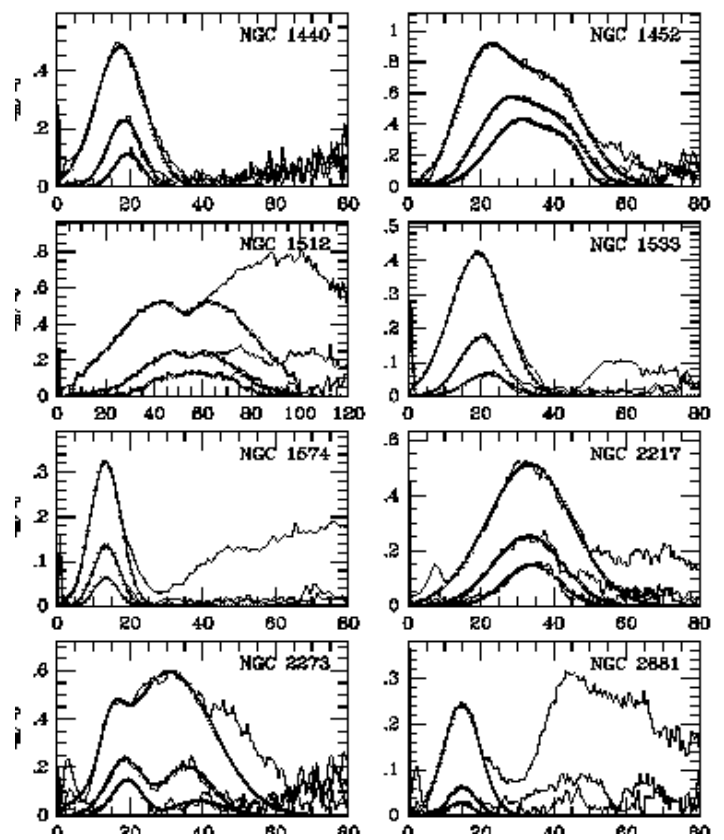
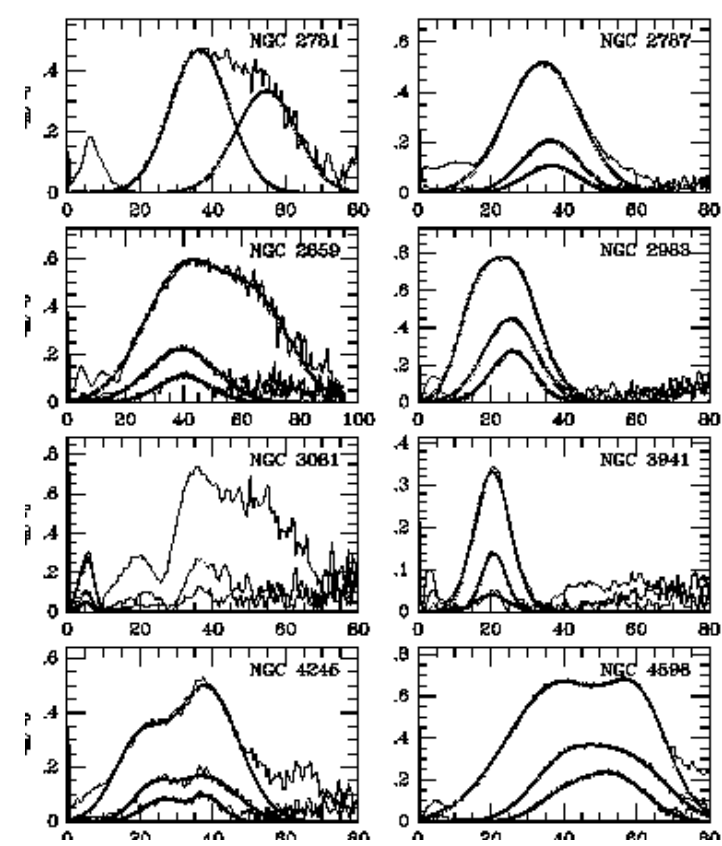

Fig. 4 (CONT.).-
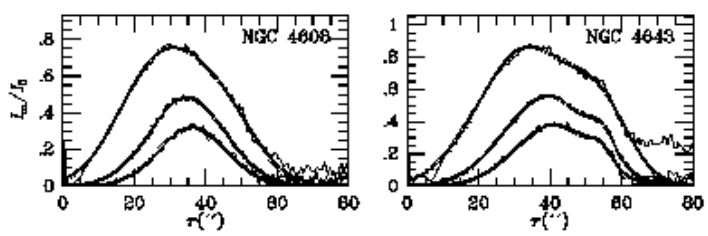

Fig. 4 (CONT.).- Gaussian representations of the $m=2$, 4 , and 6 $I_{m} / I_{0}$ profiles of the same galaxies as in Figure 3. Crosses show the fits. In NGC 1022 and 1512, the crosses are based on the symmetry assumption, not gaussian fits. For NGC 2781, the two gaussians are in slightly different position angles and are shown separately. Higher order terms than $m=6$ could also be fitted similarly but are not shown.

FIG. 4 (CONT.).- 


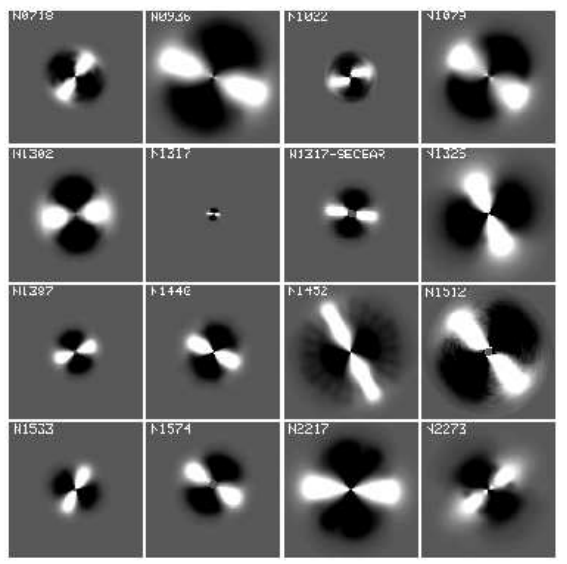

FIG. 5.-

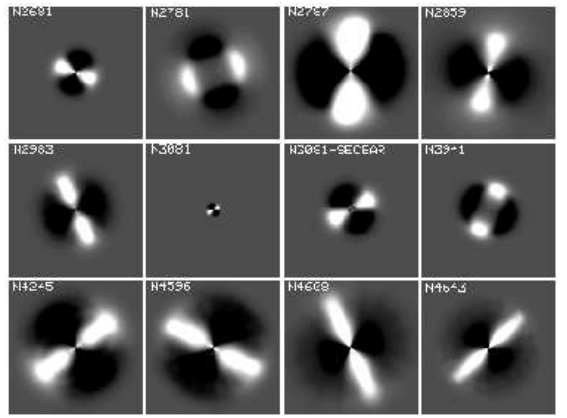

Fig. 5 (CONT.).- Reconstructed images of the bars of the 26 galaxies based on the gaussian or symmetry assumption representations shown in Figure 4. Only even Fourier terms up to a maximum $m_{\max }$ were used depending on the strength of the bar. For the strongest bars in the sample, $m_{\max }=20$. The dimensions of each square are the same as in Figure 2. 


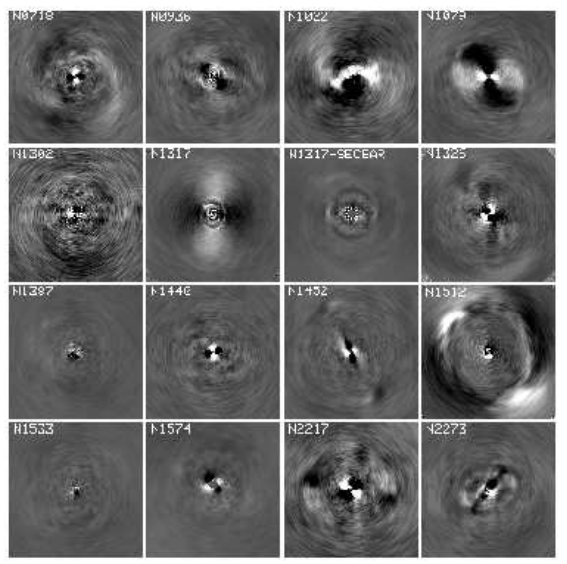

FIG. 6.-

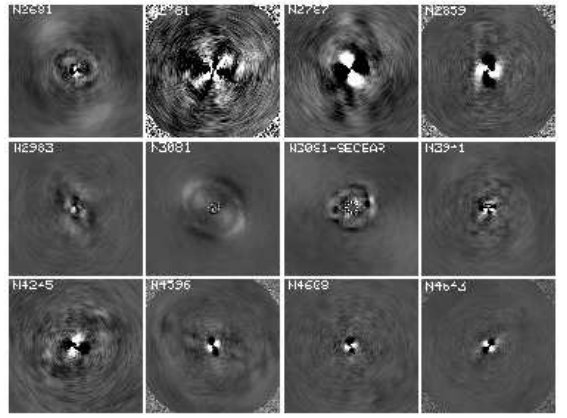

Fig. 6 (CONT.).- Residual $m>0$ images of the 26 galaxies on the same scales as in Figure 5. These include even and odd Fourier terms up to $m_{\max }=20$ as needed. These maps show asymmetries, extra bar-like features in the center, and the imperfections of some of the gaussian fits. The dimensions of each square are the same as in Figure 2. 


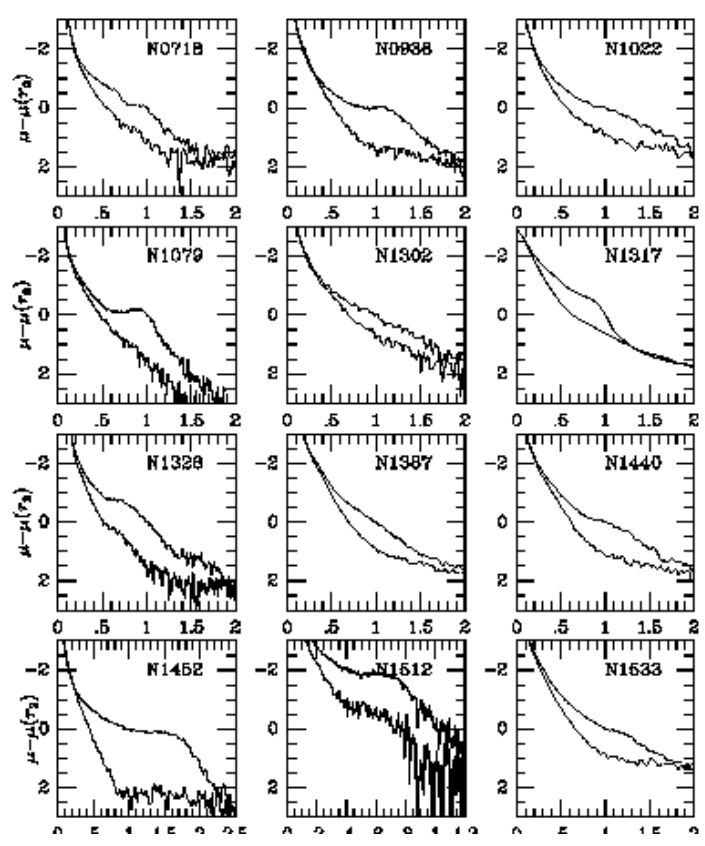

FIG. $7 .-$
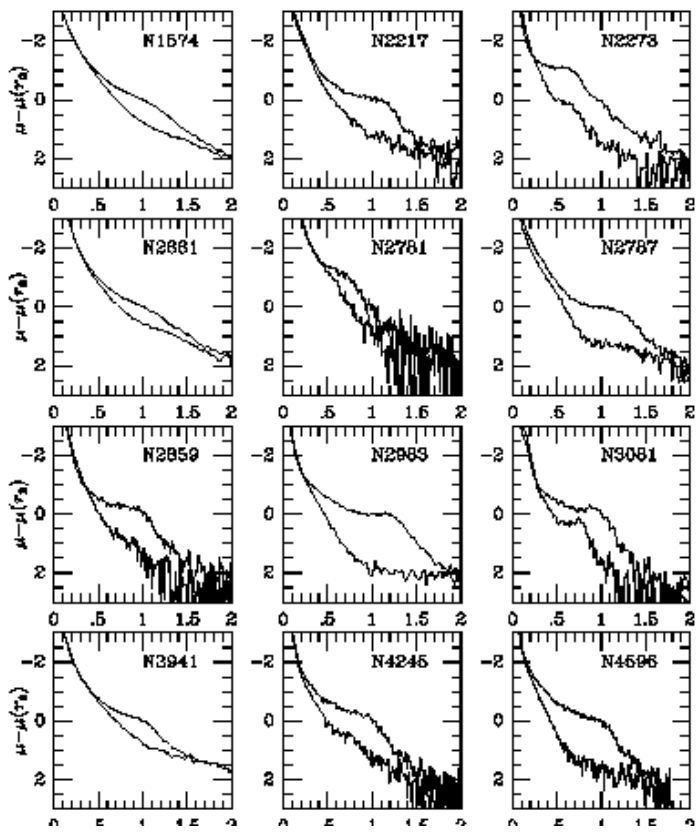

FIG. 7. 


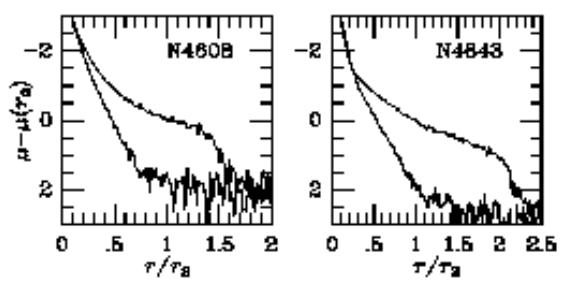

FIG. 7 (CONT.).- Radial surface brightness profiles along the major and minor axes of the main bar. The radii are normalized to the radius $r_{2}$ of the $m=2$ Fourier term, while surface brightness is shown relative to the surface brightness at this same radius. For NGC 1317, the curves shown are for the secondary bar, not the primary oval.
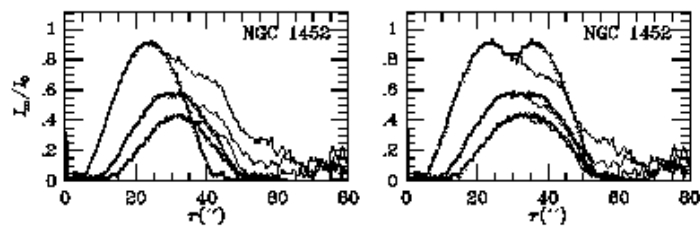

FIG. 8.- Two representations of the bar in NGC 1452 based on application of the symmetry assumption, as opposed to the gaussian fits shown in Figure 4 The left plot shows the mapping (crosses) for $r_{2}=24^{\prime \prime}$ while the right plot shows the mapping for $r_{2}=30^{\prime \prime}$. The latter resembles what we have used for NGC 1512 in Figure 4
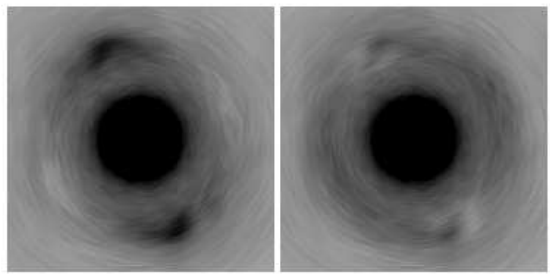

FIG. 9.- Residual intensities in NGC 1452 after removal of the bar mappings in Figure 8 The left panel is based on $r_{2}=24^{\prime \prime}$ while the right panel is for $r_{2}=30^{\prime \prime}$.

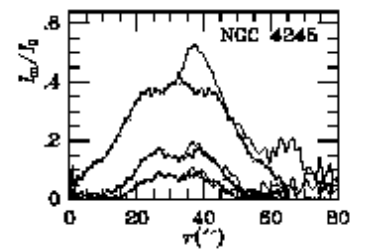

FIG. 10.- A representation of the bar in NGC 4245 based on application of the symmetry assumption (crosses), as opposed to the gaussian fits shown in Figure 4 